\title{
Higgs and dark matter physics in the type-II two-Higgs-doublet model inspired by $E_{6}$ GUT
}

\author{
P. Ko, ${ }^{a}$ Yuji Omura ${ }^{b}$ and Chaehyun $\mathbf{Y u}^{a}$ \\ a School of Physics, KIAS, \\ Seoul 130-722, Korea \\ ${ }^{b}$ Department of Physics, Nagoya University, \\ Nagoya 464-8602, Japan \\ E-mail: pko@kias.re.kr, yujiomur@eken.phys.nagoya-u.ac.jp, \\ chyu@kias.re.kr
}

ABstract: We study Higgs and dark matter physics in the type-II two-Higgs-doublet model $(2 \mathrm{HDM})$ with an extra $\mathrm{U}(1)_{H}$ gauge symmetry, inspired by the $E_{6}$ grand unified theory (GUT). From the viewpoint of the bottom-up approach, the additional $\mathrm{U}(1)_{H}$ gauge symmetry plays a crucial role in avoiding the tree-level flavor changing neutral currents mediated by neutral Higgs bosons in general $2 \mathrm{HDMs}$. In the model with $\mathrm{U}(1)_{H}$ gauge symmetry, which has Type-II Yukawa couplings, we have to introduce additional chiral fermions that are charged under the $\mathrm{U}(1)_{H}$ gauge symmetry as well as under the StandardModel (SM) gauge symmetry in order to cancel chiral gauge anomalies. For the $\mathrm{U}(1)_{H}$ charge assignment and the extra matters, we adopt the ones inspired by the $E_{6}$ GUT: the extra quark-like and lepton-like fermions with the non-trivial $\mathrm{U}(1)_{H}$ charges. We discuss their contributions to the physical observables, such as the measurements of Higgs physics and electro-weak interactions, and investigate the consistency with the experimental results. Furthermore, we could find extra neutral particles like the SM neutrinos after the electro-weak symmetry breaking, and they could be stable, because of the remnant symmetry after $\mathrm{U}(1)_{H}$ symmetry breaking. We also discuss the thermal relic density and the (in)direct-detections of this dark matter candidate.

Keywords: Higgs Physics, Beyond Standard Model, Cosmology of Theories beyond the SM

ARXIV EPRINT: 1502.00262 


\section{Contents}

1 Introduction 1

2 Type-II 2HDM with Higgs symmetry 4

2.1 Type-II $2 \mathrm{HDM}$ with gauged $\mathrm{U}(1)_{H}$ symmetry inspired by $E_{6}$ GUT 4

3 Stability of the extra particles and the dark matter candidate $\quad 7$

3.1 Extra leptons $\quad 7$

3.2 Extra quarks 8

4 Theoretical and experimental bounds $\quad 9$

4.1 Parameters 9

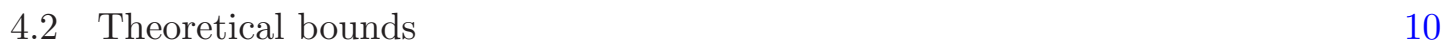

$\begin{array}{lll}4.3 & \text { Experimental constraints } & 11\end{array}$

4.3.1 Electroweak precision observables (EWPOs) 11

4.3.2 Constraints on the charged Higgs boson 12

$\begin{array}{lll}\text { 4.3.3 Constraints on the neutral (pseudo)scalar bosons } & 12\end{array}$

$\begin{array}{lll}\text { 4.3.4 } & \mathrm{U}(1)_{H} \text { gauge boson } Z_{H} & 13\end{array}$

5 Higgs signals at the LHC $\quad 14$

$\begin{array}{lll}6 & \text { Dark matter physics } & 17\end{array}$

$\begin{array}{llr}7 & \text { Summary } & 20\end{array}$

$\begin{array}{ll}\text { A Interactions of exotic fermions } & 21\end{array}$

B Contribution of the extra lepton to the vacuum polarization 22

\section{Introduction}

Adding extra Higgs doublets to the Standard Model (SM) would be one of the most attractive and the simplest ways to consider the extension of the SM. In fact, such extra Higgs doublets are present in many Beyond Standard Models (BSMs) motivated by some theoretical problems of the SM such as gauge hierarchy problem. Theoretical and phenomenological aspects of multi-Higgs-Doublet models have been widely discussed so far. Especially, two-Higgs-Doublet models (2HDM) with (softly broken) $Z_{2}$ Higgs symmetry are well-investigated, motivated by supersymmetry, grand unification theories (GUT), Higgs and dark matter physics (see ref. [1] for recent reviews). Also a lot of interests on this model have been drawn in light of new LHC data [2-9]. 
The softly broken discrete $Z_{2}$ Higgs symmetry is introduced to avoid the tree-level flavor changing neutral currents (FCNCs) á la Natural Flavor Conservation (NFC) criterion [13]. The resulting $2 \mathrm{HDMs}$ predict the so-called minimal flavor violation, where the FCNCs mediated by neutral Higgs bosons are suppressed by the CKM matrix and thus phenomenologically safe.

In order to avoid too large tree-level FCNCs, the present authors made new proposals of (flavor-dependent) gauged $\mathrm{U}(1)_{H}$ Higgs symmetry instead of the $Z_{2}$ symmetry in refs. [14-17]. In the $2 \mathrm{HDMs}$ with $\mathrm{U}(1)_{H}$ symmetry (denoted as $2 \mathrm{HDM}_{\mathrm{U}(1)}$ hereafter), two Higgs doublets are charged under new local $\mathrm{U}(1)_{H}$ gauge symmetry, and they break both electroweak $(\mathrm{EW})$ and $\mathrm{U}(1)_{H}$ Higgs gauge symmetries. In this new proposal, SM fermions have to be charged under $\mathrm{U}(1)_{H}$; otherwise one cannot write the realistic Yukawa couplings at the renormalizable level.

The $2 \mathrm{HDM}_{\mathrm{U}(1)}$ is strongly constrained by the measurements of the electroweak precision observables (EWPOs), as well as the collider searches for $Z^{\prime}$ and Higgs boson. In fact, the present authors investigated the constraints in the type-I $2 \mathrm{HDM}_{\mathrm{U}(1)}$ in detail, and discussed the current status of the Type-I $2 \mathrm{HDM}_{\mathrm{U}(1)}$ in light of the recent LHC results on Higgs properties and provided the future prospects in ref. [14]. Also they constructed the inert $2 \mathrm{HDM}$ model with $\mathrm{U}(1)_{H}$ gauge symmetry and showed that the light dark matter (DM) mass region below $\sim m_{W}$ is widely open if the $Z_{2}$ symmetry is implemented into local $\mathrm{U}(1)_{H}$ gauge symmetry, due to newly open annihilation channels of the DM pair into the extra $\mathrm{U}(1)_{H}$ gauge boson(s): $H H \rightarrow Z_{H} Z_{H}, Z Z_{H}$, which are not present in the ordinary inert $2 \mathrm{HDM}$ with discrete $Z_{2}$ symmetry [18]. In fact this phenomenon is very generic in dark matter models with local dark gauge symmetries [19-24]. Note that the $\mathrm{U}(1)_{H}$ gauge symmetry is nothing but local dark gauge symmetry, since it acts only on the inert doublet, and not to the SM fields at all.

We may have to introduce extra chiral fermions to avoid gauge anomalies depending on the $\mathrm{U}(1)_{H}$ charge assignments to the SM fermions. In ref. [14], it was shown that the anomaly-free $\mathrm{U}(1)_{H}$ charge assignments to the SM fermions are possible in the Type-I $2 \mathrm{HDM}_{\mathrm{U}(1)}$, so the fermion sector is just the same as the SM case except right-handed neutrinos. However, in other types of $2 \mathrm{HDMs}, \mathrm{U}(1)_{H}$ becomes anomalous without extra chiral fermions, and then we face the strong constraints on extra fermions from various experiments. For example, in the Type-II $2 \mathrm{HDM}_{\mathrm{U}(1)}$, which is the main subject of this work, there is no solution for the anomaly-free conditions without extra chiral fermions, as discussed in ref. [15]. The extra particles would be colored and carry the electric charges. Hence, they would be produced and detected at the LEP and hadron colliders, depending on their masses [15]. Therefore such additional particles charged under $\mathrm{U}(1)_{H}$ and/or the SM gauge groups would suffer from strong theoretical and experimental constraints. On the other hand, some of them might be stable (or long-lived enough) and could be good cold dark matter $(\mathrm{CDM})$ candidates as pointed out in refs. [15, 18]. Their stability could be guaranteed by the remnant symmetry of $\mathrm{U}(1)_{H}[18]$.

In this paper, we study Higgs and dark matter physics, as well as the experimental and theoretical constraints, in the Type-II $2 \mathrm{HDM}_{\mathrm{U}(1)}$, inspired by $E_{6}$ GUT. From the point of view of the bottom-up approach, there are many choices for the $\mathrm{U}(1)_{H}$ charge assignment 
to realize the Type-II Yukawa couplings, where one Higgs doublet couples with the up-type fermions and the other one couples with the down-type fermions in accordance with NFC. One well-known $\mathrm{U}(1)_{H}$ Higgs gauge symmetry would be the one predicted by GUT, such as $E_{6}$ and $\mathrm{SO}(10)$ GUT. The rank of $E_{6}$ gauge group is "6", so that $E_{6}$ predicts 2 extra U(1) symmetries and the SM fermions, as well as extra fermions to make the models free of gauge anomalies. They could be derived from the three-family $\mathbf{2 7}$ representations at low energy, in the supersymmetric $E_{6}$ model. ${ }^{1}$ If we assume that $\mathrm{U}(1)_{H}$ is originated from breaking of two $\mathrm{U}(1)$ symmetries at low energy scale, the $\mathrm{U}(1)_{H}$ charges are predicted explicitly by the RG flow and the decoupling scales of the extra fields $[29,30]$. The representative $\mathrm{U}(1)_{H}$ charge assignments are $\mathrm{U}(1)_{\psi}, \mathrm{U}(1)_{\chi}$, and $\mathrm{U}(1)_{\eta}$, and they face stringent constraints from the Drell-Yan (DY) processes from hadron colliders [31]. However, if we assume $\mathrm{U}(1)_{H}$ is the so-called leptophobic $\mathrm{U}(1)_{b}$ under which the SM leptons are not charged [29, 30, 32-35], one could evade the strong constraints from the Drell-Yan processes, and the $\mathrm{U}(1)_{H}$ gauge boson could be as low as $\sim O(100) \mathrm{GeV}$.

In our type-II $2 \mathrm{HDM}_{\mathrm{U}(1)}$, we shall assign one gauged $\mathrm{U}(1)_{H}$ symmetry, which may be derived from the $E_{6}$ GUT model, assuming one of the two U(1)'s is broken at a high scale. From the viewpoint of the top-down approach, the $\mathrm{U}(1)_{H}$ may be fixed once we chose the broken U(1) symmetry, because they may be approximately orthogonal each other. For instance, $\mathrm{U}(1)_{H}$ may be the linear combination of $\mathrm{U}(1)_{Y}$ and $\mathrm{U}(1)_{\psi}$ when $\mathrm{U}(1)_{\chi}$ is broken at high energy.

In this paper, we define the $\mathrm{U}(1)_{H}$ as the leptophobic $\mathrm{U}(1)_{b}$ which is the linear combination of $\mathrm{U}(1)_{\eta}$ and $\mathrm{U}(1)_{Y}$ taking the bottom-up approach. This is because we could expect that such leptophobic interaction may be sizable enough so that we may be able to observe new physics effects at colliders and dark matter experiments as demonstrated in the following. Furthermore, we consider the Yukawa couplings which respect both $\mathrm{U}(1)_{b}$ and $\mathrm{U}(1)_{\psi}$ (or $\left.\mathrm{U}(1)_{\chi}\right)$, in order to avoid the FCNCs induced by the mass mixings between extra fermions and the SM fermions. ${ }^{2}$

Besides, we introduce only two Higgs doublets and the minimal set of the chiral fermions for the anomaly-free conditions which could be coming from the three-family of fundamental 27's. The extra fermions consist of the quark-like and lepton-like particles whose charges under the SM gauge groups are the same as the right-handed down quarks, the left-handed leptons and the right-handed neutrinos. After EW symmetry breaking, the extra leptons are decomposed into neutral and charged particles just like neutrinos and charged leptons in the SM. In fact, there are 9 extra neutral and 6 charged particles, as we will see in section 2 , and we could find the lightest neutral particle among them. The extra $\mathrm{U}(1)_{H}$ symmetries are spontaneously broken but the remnant $Z_{2}^{\text {ex }}$ symmetry is conserved. The lightest particle is charged under $\mathrm{U}(1)_{H}$ and has odd parity under the remnant $Z_{2}^{\text {ex }}$ symmetry, so that it becomes a good dark matter candidate. DM will interact with SM particles through $Z_{H}$ and scalar boson exchanges.

\footnotetext{
${ }^{1}$ The general analysis of $Z^{\prime}$ in the $E_{6}$ GUT has been done in refs. [25, 26]. One can also see the reviews of the $E_{6}$ GUT [27, 28].

${ }^{2} \mathrm{U}(1)_{\psi}$ and $\mathrm{U}(1)_{\chi}$ are not orthogonal to the leptophobic $\mathrm{U}(1)_{b}$. We will also give comments on the case with $\mathrm{U}(1)_{H} \equiv \mathrm{U}(1)_{\psi}$ or $\mathrm{U}(1)_{\chi}$.
} 
This paper is organized as follows. In section 2, we introduce the setup of our $2 \mathrm{HDM}_{\mathrm{U}(1)}$, presenting the extra $\mathrm{U}(1)_{H}$ charge assignments to the SM fermions and extra chiral fermions for the anomaly cancellation. Then we discuss the interactions of the extra particles and the stability of the CDM candidate in section 3. Then, we study the contributions of the $\mathrm{U}(1)_{H}$ gauge boson and the extra fermions to the EWPOs, Higgs signals and phenomenology of CDM in section 4,5 and 6, respectively. Finally, section 7 is devoted to summary. The gauge interactions and the vacuum polarizations in our models are introduced in appendix A and B.

\section{Type-II 2HDM with Higgs symmetry}

In order to realize the minimal flavor violation, one fermion sector should couple with one Higgs doublet á la NFC. Such Yukawa couplings can be realized by assuming an additional symmetry that distinguishes the two Higgs doublets: $Z_{2}$ symmetry [13] or gauged $\mathrm{U}(1)_{H}$ symmetry [15]. In the $2 \mathrm{HDM}_{\mathrm{U}(1)}$, the $\mathrm{SM}$ particles are also charged under the additional gauge symmetry and extra chiral fermions might be required to cancel the anomaly. In ref. [14], the type-I $2 \mathrm{HDM}_{\mathrm{U}(1)}$ is mainly discussed and the gauged $\mathrm{U}(1)_{H}$ symmetry is anomaly-free without any extra chiral fermions except right-handed neutrinos. In the typeII $2 \mathrm{HDM}_{\mathrm{U}(1)}$, the anomaly-free conditions cannot be satisfied without extra fermions [15], so that we have to consider the more complex matter content and $\mathrm{U}(1)_{H}$ charge assignment. We could consider many models where the gauge anomalies are canceled by the extra fields as discussed in ref. [15]. In this section, we introduce the type-II $2 \mathrm{HDM}_{\mathrm{U}(1)}$ inspired by $E_{6}$ GUT.

\subsection{Type-II $2 \mathrm{HDM}$ with gauged $\mathrm{U}(1)_{H}$ symmetry inspired by $E_{6}$ GUT}

The scalar potential of general $2 \mathrm{HDMs}$ with $\mathrm{U}(1)_{H}$ is completely fixed by local gauge invariance and renormalizability:

$$
\begin{aligned}
V= & \hat{m}_{1}^{2}\left(|\Phi|^{2}\right) H_{1}^{\dagger} H_{1}+\hat{m}_{2}^{2}\left(|\Phi|^{2}\right) H_{2}^{\dagger} H_{2}+m_{\Phi}^{2}|\Phi|^{2}+\lambda_{\Phi}|\Phi|^{4}-\left(m_{3}^{2}(\Phi) H_{1}^{\dagger} H_{2}+\text { h.c. }\right) \\
& +\frac{\lambda_{1}}{2}\left(H_{1}^{\dagger} H_{1}\right)^{2}+\frac{\lambda_{2}}{2}\left(H_{2}^{\dagger} H_{2}\right)^{2}+\lambda_{3}\left(H_{1}^{\dagger} H_{1}\right)\left(H_{2}^{\dagger} H_{2}\right)+\lambda_{4}\left|H_{1}^{\dagger} H_{2}\right|^{2} .
\end{aligned}
$$

Here $\Phi$ is a SM singlet complex scalar field with $\mathrm{U}(1)_{H}$ charge, $q_{\Phi}$, and contributes to the $\mathrm{U}(1)_{H}$ symmetry breaking. $\hat{m}_{i}^{2}\left(|\Phi|^{2}\right)(i=1,2)$ and $m_{3}^{2}(\Phi)$ are functions of $\Phi$ only:

$$
\hat{m}_{i}^{2}\left(|\Phi|^{2}\right)=m_{i}^{2}+\widetilde{\lambda}_{i}|\Phi|^{2}
$$

at the renormalizable level. The function $m_{3}^{2}(\Phi)$ is fixed by the $\mathrm{U}(1)_{H}$ charges $\left(q_{H_{i}}\right)$ of the Higgs doublets $\left(H_{i}\right)$ and $q_{\Phi}$, and $m_{3}^{2}(\langle\Phi\rangle)=0$ is satisfied at $\langle\Phi\rangle=0: m_{3}^{2}(\Phi)=\mu \Phi^{n}$, with $n \equiv\left(q_{H_{1}}-q_{H_{2}}\right) / q_{\Phi}$. The parameter $\mu$ can be rendered real after suitable redefinition of the phase of $\Phi$. Note that the $\lambda_{5}$ term

$$
\frac{1}{2} \lambda_{5}\left[\left(H_{1}^{\dagger} H_{2}\right)^{2}+\text { h.c. }\right]
$$

in usual $2 \mathrm{HDM}$ s does not appear in the potential of our model because we employ a continuous $\mathrm{U}(1)_{H}$ gauge symmetry rather than a discrete $Z_{2}$ symmetry. 


\begin{tabular}{|c|c|c|c|c|c|c|c|}
\hline & $\mathrm{SU}(3)$ & $\mathrm{SU}(2)$ & $\mathrm{U}(1)_{Y}$ & $\mathrm{U}(1)_{b}$ & $\mathrm{U}(1)_{\psi}$ & $\mathrm{U}(1)_{\chi}$ & $\mathrm{U}(1)_{\eta}$ \\
\hline$Q^{i}$ & 3 & 2 & $1 / 6$ & $-1 / 3$ & 1 & -1 & -2 \\
\hline$U_{R}^{i}$ & 3 & 1 & $2 / 3$ & $2 / 3$ & -1 & 1 & 2 \\
\hline$D_{R}^{i}$ & 3 & 1 & $-1 / 3$ & $-1 / 3$ & -1 & -3 & -1 \\
\hline$L_{i}$ & 1 & 2 & $-1 / 2$ & 0 & 1 & 3 & 1 \\
\hline$E_{R}^{i}$ & 1 & 1 & -1 & 0 & -1 & 1 & 2 \\
\hline$N_{R}^{i}$ & 1 & 1 & 0 & 1 & -1 & 5 & 5 \\
\hline$H_{1}$ & 1 & 2 & $1 / 2$ & 0 & 2 & 2 & -1 \\
\hline$H_{2}$ & 1 & 2 & $1 / 2$ & 1 & -2 & 2 & 4 \\
\hline
\end{tabular}

Table 1. Charge assignments of the SM fermions under the SM gauge group and various U(1) subgroups of $E_{6}$ group.

The Yukawa couplings in the Type-II 2HDMs are defined as

$$
V_{y}=y_{i j}^{U}{\overline{Q_{L}}}^{i} \widetilde{H}_{2} U_{R}^{j}+y_{i j}^{D}{\overline{Q_{L}}}^{i} H_{1} D_{R}^{j}+y_{i j}^{E} \bar{L}^{i} H_{1} E_{R}^{j}+y_{i j}^{N} \bar{L}^{i} \widetilde{H}_{2} N_{R}^{j}+\text { h.c.. }
$$

Note that the $H_{1}$ and $H_{2}$ should carry different $\mathrm{U}(1)_{H}$ charges in order to distinguish these two. ${ }^{3}$ In the Type-II $2 \mathrm{HDM}$ inspired by $E_{6}$, the charge assignments for the SM particles and the Higgs doublets are given in table 1.

Let us assume that $E_{6}$ gauge symmetry breaks down as

$$
E_{6} \rightarrow \mathrm{SO}(10) \times \mathrm{U}(1)_{\psi} \rightarrow \mathrm{SU}(5) \times \mathrm{U}(1)_{\chi} \times \mathrm{U}(1)_{\psi} .
$$

The linear combination of $\mathrm{U}(1)_{\psi}$ and $\mathrm{U}(1)_{\chi}$ gives $\mathrm{U}(1)_{\eta}$, and the leptophobic $\mathrm{U}(1)_{b}$ is defined by their linear combinations with $\mathrm{U}(1)_{Y}[29,30,32-35]$ :

$$
\begin{aligned}
Q_{\eta} & =\frac{3}{4} Q_{\chi}-\frac{5}{4} Q_{\psi}, \\
Q_{b} & =\frac{1}{5}\left(Q_{\eta}+2 Q_{Y}\right) .
\end{aligned}
$$

We can see the charge assignment for each U(1) symmetry in table 1 .

The U(1) charge assignments of the SM fermions do not satisfy the anomaly-free conditions, and we have to introduce the following extra chiral fermions for anomaly cancellation:

$$
q_{L}^{i}, q_{R}^{i}, l_{L}^{i}, l_{R}^{i}, n_{L}^{i}
$$

Here $n_{L}^{i}$ is neutral, and $\left(q_{L}^{i}, q_{R}^{i}\right)$ and $\left(l_{L}^{i}, l_{R}^{i}\right)$ are vector-like fermions under the SM gauge groups. Their $\mathrm{U}(1)$ charges are chiral, as shown in table 2. The generation index, $i$, corresponds to those of the SM fermions, and anomaly-free conditions are achieved within each generation. In the $E_{6}$ GUT, the SM fermions and these extra chiral fermions are nicely embedded into three-family $\mathbf{2 7}$ representations.

\footnotetext{
${ }^{3}$ For $\mathrm{U}(1)_{\chi}$, two Higgs doublets carry the same charges, but right-handed up-type and down-type quarks have the different $\mathrm{U}(1)_{\chi}$ charges.
} 


\begin{tabular}{|c|c|c|c|c|c|c|c|}
\hline & $\mathrm{SU}(3)$ & $\mathrm{SU}(2)$ & $\mathrm{U}(1)_{Y}$ & $\mathrm{U}(1)_{b}$ & $\mathrm{U}(1)_{\psi}$ & $\mathrm{U}(1)_{\chi}$ & $\mathrm{U}(1)_{\eta}$ \\
\hline$q_{L}^{i}$ & 3 & 1 & $-1 / 3$ & $2 / 3$ & -2 & 2 & 4 \\
\hline$q_{R}^{i}$ & 3 & 1 & $-1 / 3$ & $-1 / 3$ & 2 & 2 & -1 \\
\hline$l_{L}^{i}$ & 1 & 2 & $-1 / 2$ & 0 & -2 & -2 & 1 \\
\hline$l_{R}^{i}$ & 1 & 2 & $-1 / 2$ & -1 & 2 & -2 & -4 \\
\hline$n_{L}^{i}$ & 1 & 1 & 0 & -1 & 4 & 0 & -5 \\
\hline
\end{tabular}

Table 2. Charge assignments of the exotic chiral fermions under the SM gauge group and various $\mathrm{U}(1)$ subgroups of $E_{6}$.

\begin{tabular}{|c|c|c|c|c|c|c|c|}
\hline & $\mathrm{SU}(3)$ & $\mathrm{SU}(2)$ & $\mathrm{U}(1)_{Y}$ & $\mathrm{U}(1)_{b}$ & $\mathrm{U}(1)_{\psi}$ & $\mathrm{U}(1)_{\chi}$ & $\mathrm{U}(1)_{\eta}$ \\
\hline$\Phi$ & 1 & 1 & 0 & 1 & -4 & 0 & 5 \\
\hline
\end{tabular}

Table 3. Charge assignments of a singlet scalar $\Phi$ under the SM gauge group and U(1) subgroup of $E_{6}$. This scalar $\Phi$ makes an additional contribution to $U(1)$ symmetry breaking.

We have also introduced one extra complex scalar, $\Phi$, which is a singlet under the SM gauge group, in order to break $\mathrm{U}(1)_{H}$ spontaneously and generate the mass terms of the extra fermions. Let us define the charges of $\Phi$ as shown in table $3{ }^{4}$ Then the Yukawa couplings which respect all extra $\mathrm{U}(1)$ symmetries are given by

$$
V_{y}^{\mathrm{ex}}=y_{i j}^{q} \Phi{\overline{q_{L}}}^{i} q_{R}^{j}+y_{i j}^{l} \Phi{\overline{\bar{l}_{L}}}^{i} l_{R}^{j}+y_{i j}^{n}{\overline{l_{R}}}^{i} \widetilde{H}_{1} n_{L}^{j}+y_{i j}^{\prime n}{\overline{l_{L}^{c}}}^{i} H_{2} n_{L}^{j}+\text { h.c.. }
$$

When $\Phi$ develops a nonzero vacuum expectation value (VEV), $q_{L, R}^{i}$ and $l_{L, R}^{i}$ would become massive through the Yukawa couplings.

As we discussed in section 1 , we assume only one $\mathrm{U}(1)_{H}$ gauge symmetry, which is the linear combination of the all U(1) symmetries and remains at low energy, whereas the other $\mathrm{U}(1)$ from $E_{6}$ is spontaneously broken at the high energy scale. If $\mathrm{U}(1)_{\psi}$ or $\mathrm{U}(1)_{\chi}$ is broken, the following Yukawa couplings would be allowed,

$$
V_{y}^{\mathrm{FCNC}}=c_{i j}^{D} \Phi{\overline{q_{L}}}^{i} D_{R}^{j}+c_{i j}^{L} \Phi \bar{L}^{i} l_{R}^{j}+c_{i j}^{Q}{\overline{Q_{L}}}^{i} q_{R}^{j} H_{1}+c_{i j}^{E}{\overline{\bar{l}_{L}}}^{i} E_{R}^{j} H_{1}+c_{i j}^{N}{\overline{l_{L}}}^{i} N_{R}^{j} \widetilde{H}_{2}+\text { h.c. },
$$

and the extra charged fermions mix with the SM quarks, and charged leptons and tree-level FCNC interactions will appear in general. Hence we simply assume that $\mathrm{U}(1)_{\psi}$ or $\mathrm{U}(1)_{\chi}$ is broken at some energy, but the remnant symmetry of $\mathrm{U}(1)_{\psi}$ or $\mathrm{U}(1)_{\chi}$ still holds down to low energy scale to suppress the FCNCs. In fact, $\mathrm{U}(1)_{\psi}\left(\mathrm{U}(1)_{\chi}\right)$ breaks to $Z_{2}^{\psi}\left(Z_{2}^{\chi}\right)$, if only the VEVs of $H_{1,2}$ and $\Phi$ break the U(1) symmetry. The SM fermions are even and the extra fermions are odd under the remnant $Z_{2}$ symmetry, so that the mass-mixing terms between the SM and the extra fermions in eq. (2.8) are forbidden. We could also consider the case that $\mathrm{U}(1)_{H}$ is identical to $\mathrm{U}(1)_{\psi}$, for instance, and then the Yukawa couplings in eq. (2.8) could be forbidden. In the sections for phenomenology, we adopt the $\mathrm{U}(1)_{b}$ as the $\mathrm{U}(1)_{H}$, and investigate the impact of the new interaction inspired by $E_{6}$ GUT, so that we simply assume that the mass mixings are forbidden by $Z_{2}^{\psi}\left(Z_{2}^{\chi}\right)$ symmetry at that time. We shall also give a comment on the $\mathrm{U}(1)_{H} \equiv \mathrm{U}(1)_{\psi}$ case.

\footnotetext{
${ }^{4}$ In the supersymmetric $E_{6}$ model, $\Phi^{*}$ could be interpreted as the superpartner of $n_{L}$.
} 


\section{Stability of the extra particles and the dark matter candidate}

In this section, we briefly summarize the mass spectrum of the extra chiral fermions and discuss their stability.

\subsection{Extra leptons}

Additional chiral fermions, $l_{I}^{i}(I=L, R)$ and $n_{L}^{i}$, are color-singlets and their SM charge assignment is the same as the one of the SM leptons and right-handed neutrino. After the EW symmetry breaking, a doublet $l_{I}^{i}$ would split into a charged and a neutral fermions like the SM left-handed lepton doublets. The charged fermions become massive due to the nonzero $\langle\Phi\rangle$, and the masses of the neutral fermions and $n_{L}^{i}$ are given by $\langle\Phi\rangle$ and $\left\langle H_{1,2}\right\rangle$ following eq. (2.7). The mass matrix for $l_{I}^{T}=\left(\widetilde{\nu}_{I}, \widetilde{e}_{I}\right)^{T}$ and $n_{L}$ is

$$
\begin{aligned}
\mathcal{L}_{\nu} & =-\frac{1}{2}\left(\overline{\widetilde{\nu}_{L}^{c}} \overline{\widetilde{\nu}_{R}} \overline{n_{L}^{c}}\right)\left(\begin{array}{ccc}
0 & m_{\widetilde{e}} & m_{M} \\
m_{\widetilde{e}} & 0 & m_{D} \\
m_{M} & m_{D} & 0
\end{array}\right)\left(\begin{array}{c}
\widetilde{\nu}_{L} \\
\widetilde{\nu}_{R}^{c} \\
n_{L}
\end{array}\right)+h . c . \\
& =-\frac{1}{2}\left(\overline{N_{1}} \overline{N_{2}} \overline{N_{3}}\right)\left(\begin{array}{ccc}
m_{1} & 0 & 0 \\
0 & m_{2} & 0 \\
0 & 0 & m_{3}
\end{array}\right)\left(\begin{array}{l}
N_{1} \\
N_{2} \\
N_{3}
\end{array}\right) .
\end{aligned}
$$

Each element is defined as $m_{\widetilde{e}}=y^{l} v_{\Phi} / \sqrt{2}, m_{D}=y^{n} v \cos \beta / \sqrt{2}$, and $m_{M}=y^{\prime n} v \sin \beta / \sqrt{2}$. $m_{\widetilde{e}}$ is the mass of the charged fermion, $\widetilde{e}$. In general, $m_{\widetilde{e}}, m_{D}$, and $m_{M}$ are $3 \times 3$ matrices in flavor space, and would not be diagonal. In the following, we simply assume that dimensionless constants in eq. (2.7) are flavor-blind and we omit the flavor index $i$ in $m_{\widetilde{e}}$, $m_{D}$, and $m_{M}$. At present, studying more general cases would be beyond the scope of this paper, lacking any direct evidence of new particles at the LHC.

When $\left(m_{\widetilde{e}}^{2}+m_{D}^{2}+m_{M}^{2}\right)^{3}-27\left(m_{\widetilde{e}} m_{D} m_{M}\right)^{2} \geq 0$ is satisfied, the mass eigenvalues, $m_{1}$, $m_{2}$ and $m_{3}$, are given by

$$
2 \sqrt{\frac{m_{\widetilde{e}}^{2}+m_{D}^{2}+m_{M}^{2}}{3}} \cos \frac{\theta}{3}, 2 \sqrt{\frac{m_{\widetilde{e}}^{2}+m_{D}^{2}+m_{M}^{2}}{3}} \cos \left(\frac{\theta}{3} \pm \frac{2 \pi}{3}\right),
$$

where $\theta$ is defined as

$$
\tan \theta=\frac{\sqrt{\left\{\left(m_{\widetilde{e}}^{2}+m_{D}^{2}+m_{M}^{2}\right) / 3\right\}^{3}-\left(m_{\widetilde{e}} m_{D} m_{M}\right)^{2}}}{\left(m_{\widetilde{e}} m_{D} m_{M}\right)} .
$$

Three eigenstates of neutral fermions, $\left(N_{1}, N_{2}, N_{3}\right)$, are linear combinations of $\widetilde{\nu}_{L}, \widetilde{\nu}_{L}^{c}, n_{L}$, defined as

$$
\left(\begin{array}{c}
\widetilde{\nu}_{L} \\
\widetilde{\nu}_{R}^{c} \\
n_{L}
\end{array}\right)=\sum_{a=1}^{3} \frac{1}{\sqrt{1+\left(\frac{m_{D}^{2}-m_{a}^{2}}{m_{D} m_{\tilde{e}}+m_{M} m_{a}}\right)^{2}+\left(\frac{m_{D} m_{M}+m_{\tilde{e}} m_{a}}{m_{D} m_{\tilde{e}}+m_{M} m_{a}}\right)^{2}}}\left(\begin{array}{c}
-\frac{m_{D}^{2}-m_{a}^{2}}{m_{D} m_{\tilde{e}}+m_{M} m_{a}} \\
\frac{m_{D} m_{M}+m_{\tilde{e}} m_{a}}{m_{D} m_{\tilde{e}}+m_{M} m_{a}} \\
1
\end{array}\right) P_{L} N_{a}
$$

where $P_{L}$ is the projection operator, $P_{L}=\left(1-\gamma_{5}\right) / 2$. 
Defining $Z_{2}^{\mathrm{ex}} \equiv Z_{2}^{\psi} \times(-1)^{2 s}$ or $Z_{2}^{\chi} \times(-1)^{2 s}$ with $s$ being the spin of the particle, we can assign the odd $Z_{2}^{\text {ex }}$ charge to all the exotic fermions. This remnant $Z_{2}$ symmetry guarantees the stability of the lightest particle among the exotic fermions, so that the lightest neutral particle $(\equiv X)$ among $N_{a}$ could be a good cold dark matter candidate.

The extra charged lepton, $\widetilde{e}$, should decay in order to avoid a stable charged particle. After the EW symmetry breaking, the gauge interactions of $\widetilde{e}$ and $N_{a}$ are described in appendix A. If $\widetilde{e}$ is heavier than at least $X$, the charged exotic lepton $\widetilde{e}$ decays to the DM $X$ and the SM fermions through the $W^{(*)}$ exchange.

The exotic leptons $\tilde{e}$ and $N_{a}$ can be produced at colliders by DY processes through the $s$-channel $W^{ \pm}, \gamma$ and/or $Z^{0}, Z_{H}$ exchanges. Note that the DY process through $Z_{H}$ exchange is a new aspect in our model, since $Z_{H}$ couples both to the SM quarks and exotic fermions. Once they are produced at colliders, they will decay through the mixing in eq. (A.1) (and the higher-dimensional operators), the extra leptons and quarks decay as

$$
N_{a=2,3} \rightarrow X+Z^{0(*)}, \quad \widetilde{e}^{ \pm} \rightarrow N_{a=1,2,3}+W^{ \pm(*)},
$$

and $W^{ \pm}$or $Z^{0}$ (either real or virtual) will decay into two SM fermions. Therefore, their collider signatures would be similar to those of charginos and neutralinos in supersymmetric models, and bounds on chargino and neutralinos could be applied to our model with simple modification. The lower bound on $m_{\widetilde{e}}$ would be around $800 \mathrm{GeV}$, inferred from $p p \rightarrow \chi^{ \pm} \chi^{0}$, $\chi^{ \pm} \chi^{ \pm}[36,37]$.

\subsection{Extra quarks}

The SM charges of extra quarks, $q_{I}^{i}(I=L, R)$, are the same as those of the right-handed down quarks, $D_{R}^{i}$. However they can be distinguished by the $\mathrm{U}(1)_{H}$ charges and $Z_{2}^{\text {ex }}$. In fact, the mass mixing between the extra quarks and the SM quarks in eq. (2.8) is forbidden by the symmetry, so that the tree-level FCNCs involving the extra quarks are absent and the exotic quarks can not decay at the renormalizable level, in the $2 \mathrm{HDM}_{\mathrm{U}(1)}$ with $Z_{2}^{\text {ex }}$. There might be $Z_{2}^{\text {ex }}$ symmetric higher-dimensional operators. For example, a dim-8 operator such as

$$
\frac{c_{i j k l}}{\Lambda^{4}} \Phi{\overline{q_{L}}}^{i} D_{R}^{j}{\overline{l_{L}}}^{k} E_{R}^{l} H_{1}+\text { h.c. }
$$

would make the extra quarks decay into the SM fermions and the DM $X$, with the decay width given by

$$
\Gamma \sim \frac{1}{(4 \pi)^{3}}\left(\frac{v \cos \beta v_{\Phi} m_{q}^{2}}{\Lambda^{4}}\right)^{2} m_{q},
$$

where $m_{q}$ is the mass of the exotic quark. Assuming $v_{\Phi}=m_{q}=1 \mathrm{TeV}$ and $\tan \beta \approx 1$, the lifetime is estimated as $\sim 1 \mu \mathrm{sec}$ at $\Lambda=100 \mathrm{TeV}$, which is much longer than the QCD time scale $\tau_{\mathrm{QCD}} \sim \Lambda_{\mathrm{QCD}}^{-1}$. Therefore they will be hadronized, forming exotic massive hadrons, and would decay inside or outside the detector, depending on its velocity. Thus these exotic quarks would be constrained by exotic massive particle searches. In case they decay inside the detector, the usual bounds from squark search will apply.

Exotic quarks $q_{L}^{i}$ and $q_{R}^{i}$ are produced copiously by QCD processes at hadron colliders, and by the DY process at lepton colliders. Once they are produced, they will decay through 
$Z_{2}^{\text {ex }}$ symmetric higher dimensional operators such as eq. (3.7). The extra-quark production could be constrained by the search for squark at LHC [38], and the lower bound would be also around $1 \mathrm{TeV}$. Once they are produced at colliders, they will decay into the exotic leptons and the SM quarks through the mixing in eq. (3.7), and then the exotic lepton decays into $l_{\mathrm{SM}} X$ through the gauge interaction in eq. (A.1):

$$
q_{I}^{i} \rightarrow q_{\mathrm{SM}} l_{\mathrm{SM}} \widetilde{e} \text { followed by } \quad \widetilde{e}^{ \pm} \rightarrow N_{a=1,2,3}+W^{ \pm(*)},
$$

where $q_{S M}$ and $l_{S M}$ are the SM quarks and leptons. Therefore the collider signatures will be $4 l+2 j+\mathbb{E}_{T}, 3 l+4 j+\mathbb{E}_{T}$, or $2 l+6 j+\mathbb{E}_{T}$.

\section{Theoretical and experimental bounds}

In this section, we discuss theoretical and experimental constraints on the 2HDM.

\subsection{Parameters}

In this subsection, we list the parameters in our model which will be scanned over. In the Higgs potential eq. (2.2), there are 11 parameters, $m_{i}^{2}(i=1,2, \Phi), \lambda_{j}(j=1,2,3,4, \Phi)$, $\tilde{\lambda}_{k}(k=1,2)$, and $\mu$, two of which are fixed by the mass of the SM-like Higgs boson $(h)$, $m_{h}=125 \mathrm{GeV}$, and $v=\sqrt{v_{1}^{2}+v_{2}^{2}}=246 \mathrm{GeV}$. In the numerical analysis we trace these parameters in the Higgs potential with more physical ones related with observables such as masses and the mixing angles:

- $\tan \beta=v_{2} / v_{1}$, where $v_{1}$ and $v_{2}$ are VEVs of $H_{i}$,

- $m_{A}$ : the mass of the pseudoscalar boson,

- $m_{\tilde{h}}$ : the mass of the additional neutral Higgs boson due to introducing a new scalar $\Phi$,

- $m_{H^{+}}$: the mass of the charged Higgs boson,

- $\Delta m_{H}=m_{H}-m_{A}$ : the mass difference between $H$ and $A$,

- $\alpha, \alpha_{1}, \alpha_{2}$ : the mixing angles between three neutral scalar bosons,

- $M_{Z_{H}}$ : the mass of the $\mathrm{U}(1)_{H}$ gauge boson.

The parameters in the Higgs potential can be obtained in terms of these 9 physical parameters. For example, $h, H$, and $\tilde{h}$ are the physical neutral Higgs bosons, which are mixtures of three neutral Higgs components of $H_{1}, H_{2}$, and $\Phi$, with the mixing angles $\alpha, \alpha_{1}, \alpha_{2}$. The explicit relations are shown in ref. [14]. The ranges of the parameters are chosen as $1 \leq \tan \beta \leq 100,125 \mathrm{GeV} \leq m_{A}, m_{\tilde{h}} \leq 1 \mathrm{TeV}, 360 \mathrm{GeV} \leq m_{H^{+}} \leq 1 \mathrm{TeV}$, $\left|\Delta m_{H}\right| \leq 500 \mathrm{GeV},\left|\alpha, \alpha_{1}, \alpha_{2}\right| \leq \pi / 2$, and $125 \mathrm{GeV} \leq M_{Z_{H}} \leq 1 \mathrm{TeV}$.

$\mathrm{U}(1)_{H}$ gauge interaction is parameterized by the gauge coupling $g_{H}$ and the $\mathrm{U}(1)_{H}$ gauge boson mass $\left(M_{Z_{H}}\right)$. The range of $g_{H}$ is taken to be $0 \leq g_{H} \leq 1$ because small $g_{H}$ is 
preferred due to the constraints from EWPOs as we will discuss later. We note that the VEV of $\Phi$ is obtained by

$$
v_{\phi}=\sqrt{M_{Z_{H}}^{2} / g_{H}^{2}-v^{2} \sin ^{2} \beta} .
$$

Note that the $Z_{H}$ mass is bounded from below by

$$
M_{Z_{H}} \geq g_{H} v|\sin \beta| .
$$

In the Yukawa sector for extra chiral fermions, there are four parameters, $y^{q}, y^{l}, y^{n}$, and $y^{\prime n}$ as shown in eq. (2.7). The Yukawa couplings are taken to be in the range,

$$
0 \leq y^{q}, y^{l}, y^{n}, y^{\prime n} \leq 4 \pi
$$

The masses of extra chiral fermions can be calculated in terms of the Yukawa couplings $\left(y^{q}, y^{l}, y^{n}, y^{\prime n}\right)$ and 3 scalar VEVs $\left(v_{i}\right.$ and $\left.v_{\phi}\right)$.

In summary, we scan 14 parameters in the numerical analysis in order to find the regions that are consistent with theoretical and experimental constraints. The mass of the candidate for CDM is not restricted in the discussion on Higgs physics or electroweak precision tests in sections 4.3 and 5 .

\subsection{Theoretical bounds}

In the analysis, we impose perturbativity bounds on dimensionless quartic couplings in the Higgs potential, $\left|\lambda_{i}\right| \leq k$, which are required for the model to be stable under higher-order corrections. Here $k$ is a certain number and chosen as $4 \pi$ in this work. The $2 \rightarrow 2$ scattering processes for scalar and gauge bosons are dominated by the induced quartic couplings $Q_{i}$ at very high energy while the amplitudes including triple gauge couplings are suppressed. We impose the perturbative unitarity condition on the induced quartic couplings at the tree level with $\left|Q_{i}\right| \leq 8 \pi[39-41]$.

Finally, we impose the vacuum stability bounds at the tree level, which require that the dimensionless couplings $\lambda_{1,2,3,4}$ are to satisfy the following conditions:

$$
\lambda_{1}>0, \quad \lambda_{2}>0, \quad \lambda_{3}>-\sqrt{\lambda_{1} \lambda_{2}}, \quad \lambda_{3}+\lambda_{4}>-\sqrt{\lambda_{1} \lambda_{2}},
$$

in the $\langle\Phi\rangle=0$ direction. They correspond to the ones in the usual $2 \mathrm{HDMs}$ without $\lambda_{5}$. It is noticeable that the conditions in eq. (4.2) lead the scalar mass relation

$$
m_{h}^{2}+m_{H}^{2}-m_{A}^{2}>0 .
$$

In the ordinary $2 \mathrm{HDMs}$ with softly broken $Z_{2}$ symmetry, sizable $\lambda_{5}$ is allowed and the conditions (4.2) and (4.3) should be modified by the replacements, $m_{A} \rightarrow m_{A}+\lambda_{5} v^{2}$ and $\lambda_{4} \rightarrow \lambda_{4}+\lambda_{5}$ in eqs. (4.2), and (4.3).

In the $\langle\Phi\rangle \neq 0$ direction, the vacuum-stability conditions for $\lambda_{\Phi}, \widetilde{\lambda_{1}}$ and $\widetilde{\lambda_{2}}$ are

$$
\lambda_{\Phi}>0, \lambda_{1}>\frac{\widetilde{\lambda}_{1}^{2}}{\lambda_{\Phi}}, \lambda_{2}>\frac{{\widetilde{\lambda_{2}}}_{2}^{2}}{\lambda_{\Phi}}, \lambda_{3}-\frac{\widetilde{\lambda_{1}} \widetilde{\lambda_{2}}}{\lambda_{\Phi}}>-\sqrt{\left(\lambda_{1}-\frac{{\widetilde{\lambda_{1}}}^{2}}{\lambda_{\Phi}}\right)\left(\lambda_{2}-\frac{{\widetilde{\lambda_{2}}}^{2}}{\lambda_{\Phi}}\right)},
$$




$$
\lambda_{3}+\lambda_{4}-\frac{\widetilde{\lambda_{1}} \widetilde{\lambda_{2}}}{\lambda_{\Phi}}>-\sqrt{\left(\lambda_{1}-\frac{{\widetilde{\lambda_{1}}}^{2}}{\lambda_{\Phi}}\right)\left(\lambda_{2}-\frac{{\widetilde{\lambda_{2}}}^{2}}{\lambda_{\Phi}}\right)},
$$

where the directions of $H_{1}$ and $H_{2}$ fields in the last four conditions are the same as those of $H_{1}$ and $H_{2}$ fields in eq. (4.2).

\subsection{Experimental constraints}

In this subsection, we discuss various experimental constraints on our 2HDM from collider experiments, flavor physics and the electroweak precision observables (EWPOs).

\subsubsection{Electroweak precision observables (EWPOs)}

In order to evaluate the allowed region for the new physics contributions to the EWPOs, Peskin-Takeuchi parameters $S, T$, and $U$ are often used [42], whose definitions can be found in ref. [43]. According to the recent LHC results, the bounds on $S, T$, and $U$ parameters are given by $S=0.03 \pm 0.10, T=0.05 \pm 0.12, U=0.03 \pm 0.10$, with $m_{h}^{\text {ref }}=126 \mathrm{GeV}$ and $m_{t}^{\text {ref }}=173 \mathrm{GeV}[44,45]$. The correlation coefficients are $+0.89_{S T},-0.54_{S U}$, and $-0.83 T U .{ }^{5}$ The Peskin-Takeuchi parameters have been calculated in the 2HDM (with extra scalars) [46-49] and in the $2 \mathrm{HDM}_{\mathrm{U}(1)}$ (with extra scalars and $\mathrm{U}(1)$ gauge boson) [14].

In addition to the contributions of extra scalar bosons and $\mathrm{U}(1)_{H}$ gauge boson, there may exist additional contributions from extra fermions in the type-II $2 \mathrm{HDM}_{\mathrm{U}(1)}$. Since the extra quarks are $\mathrm{SU}(3) \times \mathrm{U}(1)_{Y}$ vector-like and $\mathrm{SU}(2)$ singlet, they do not contribute to the EWPOs. The extra charged leptons and two of three-type neutral leptons are $\mathrm{SU}(2) \times \mathrm{U}(1)_{Y}$ vector-like, while the other $n_{L}$ is $\mathrm{SU}(2) \times \mathrm{U}(1)_{Y}$ singlet. The extra neutral leptons mix with each other (see section 3.1), and they contribute to the EWPOs. The detail of the extra contribution to the vacuum polarization is shown in appendix B.

As discussed in ref. [14], $Z_{H}$ contributes to the EWPOs at tree level through the mass mixing between $Z_{H}$ and $Z$, because the Higgs doublet charged under $\mathrm{U}(1)_{H}$ breaks not only the EW symmetry but also the $\mathrm{U}(1)_{H}$ symmetry. The present authors discussed the $Z_{H}$ correction to the EWPOs up to the one-loop level in ref. [14]. It is found that the $\mathrm{U}(1)_{H}$ gauge coupling $\left(g_{H}\right)$ and the gauge boson mass $\left(M_{Z_{H}}\right)$ are strictly constrained in the low $Z_{H}$ mass region especially around the $Z$ boson mass.

In the usual 2HDM, there are two massive CP-even scalars, one massive CP-odd scalar, and one charged Higgs pair after the EW symmetry breaking [1]. They contribute to the EWPOs at the one-loop level, and it is found that the mass differences among the extra scalars are especially constrained strongly [14, 46, 47].

In the $2 \mathrm{HDM}_{\mathrm{U}(1)}$, there is another extra neutral scalar $\tilde{h}$. In total, there are three neutral scalar Higgs bosons, $h, H$, and $\tilde{h}$ plus one pseudoscalar boson $A$, where $h$ is the SM-like Higgs boson [14] which has been observed at the LHC. All scalar bosons contribute to the EWPOs at the one-loop level. The $\mathrm{U}(1)_{H}$ gauge boson $\left(Z_{H}\right)$ also contributes to the EWPOs, but its contribution appears even at the tree level through the mixing between the $\hat{Z}$ and $\hat{Z}_{H}$ mixing, where $\hat{Z}$ and $\hat{Z}_{H}$ are gauge eigenstates while $Z$ and $Z_{H}$ are mass eigenstates, respectively. In the type-I $2 \mathrm{HDM}_{\mathrm{U}(1)}$, the contribution of extra scalars and

\footnotetext{
${ }^{5}$ Fixing $U=0, S=0.05 \pm 0.09$ and $T=0.08 \pm 0.07$ with the correlation coefficient +0.91 .
} 
$Z_{H}$ is discussed in ref. [14]. In the type-II case, the correction of the scalars and $Z_{H}$ boson to the EWPOs is the same as in the type-I case up to the one loop level, but there are additional contributions from the extra chiral fermion loops. They may affect the EWPOs through the self-energy diagrams of the SM gauge bosons. The formulas for such extra contributions to the EWPOs will be given in appendix B with detailed analysis.

\subsubsection{Constraints on the charged Higgs boson}

The charged Higgs boson is constrained by direct production channels in many experiments. In the type-II $2 \mathrm{HDM}$ case, the lower bound for the mass of the charged Higgs boson is about $80 \mathrm{GeV}$ at the $95 \%$ C.L. [50]. At the LHC, the stringent bound for $m_{H^{+}}$comes from search for the charged Higgs boson in the top quark decay for $m_{H^{+}}<m_{t}$ and from the direct production of the charged Higgs boson with subsequent decays $H^{+} \rightarrow \tau \nu$ or $H^{+} \rightarrow t \bar{b}$ for $m_{H^{+}}>m_{t}[51,52]$. It is found that the large $\tan \beta$ region is strongly constrained for $m_{H^{+}} \lesssim 300 \mathrm{GeV}$ from the LHC experiments.

The most stringent bound for $m_{H^{+}}$comes from flavour physics, in particular, $b \rightarrow s \gamma$ decays. In the type-II $2 \mathrm{HDM}$, the region of $m_{H^{+}} \geq 360 \mathrm{GeV}$ is allowed at $95 \%$ C.L. [53]. We adopt this bound in this work. The $B \rightarrow \tau \nu$ decays may constrain $\tan \beta$ and $m_{H^{+}}$in the type-II 2HDM. We impose the condition on the branching ratio for $B \rightarrow \tau \nu$ decays, $0.447 \times$ $10^{-4} \leq \operatorname{Br}(B \rightarrow \tau \nu) \leq 1.012 \times 10^{-4}$, which was measured at the Belle with hadronic tagging for the $\tau$ decay [54]. The other measurements for the branching ratios for $B \rightarrow \tau \nu$ decays at the Belle and the BABAR have much larger uncertainties than the above value $[55,56]$. We note that the results in this work do not change so much even though we use other results or the average of all results. The $B_{q}-\bar{B}_{q}$ mixing is also affected by the charged Higgs exchange. It is known that the $B_{q}-\bar{B}_{q}$ mixing disfavors a small $\tan \beta$ region so that we impose $\tan \beta \geq 1$ [57]. The mass of the pseudoscalar boson and $\tan \beta$ are constrained by the production of the pseudoscalar with the subsequent decays into $A \rightarrow \tau^{+} \tau^{-}$or $A \rightarrow \mu^{+} \mu^{-}[58,59]$. We take into account this constraint on $m_{A}$ and $\tan \beta$ in our analysis.

Another interesting measurement which may strongly affect the constraints on $\tan \beta$ and $m_{H^{+}}$is the branching ratio for the semileptonic decay $B \rightarrow D^{(*)} \tau \nu$. The BABAR measurement for this branching ratio indicates that the SM as well as the type-II 2HDM would be excluded with $99.8 \%$ probability [60]. This problem would require breaking of the so-called Natural Flavor Conservation criteria, which could be realized in the flavordependent $\mathrm{U}(1)$ model [61]. However, this breaking cannot be achieved in the $2 \mathrm{HDM}_{\mathrm{U}(1)}$ and the anomaly cannot be accommodated with this model. We ignore the experimental constraint from $\operatorname{Br}\left(B \rightarrow D^{(*)} \tau \nu\right)$ at the BABAR.

\subsubsection{Constraints on the neutral (pseudo)scalar bosons}

The search for the SM-like heavy Higgs boson would strongly constrains, in particular, the heavy Higgs boson mass and its couplings. The main channels for the SM-like heavy Higgs boson search are the $H \rightarrow Z Z \rightarrow 4 l$ decays in the vector boson fusion (VHF) and vector boson associated production $(\mathrm{VH})$ or in the $g g$ fusion process $(g g)$. We impose the upper bound on the signal strength $(\mu)$ for a heavy Higgs boson production and decay: $\mu_{\mathrm{VHF}+\mathrm{VH}}^{Z Z}, \mu_{\mathrm{gg}}^{Z Z} \lesssim 0.1 \sim 1$ for $125 \mathrm{GeV}<m_{H}<1 \mathrm{TeV}[62]$. 


\begin{tabular}{|c|c|c|}
\hline Higgs tagging channels & ATLAS & CMS \\
\hline$H \rightarrow \gamma \gamma$ & $1.57_{-0.28}^{+0.33}$ & $1.13 \pm 0.24$ \\
$H \rightarrow Z Z^{*}$ & $1.44_{-0.35}^{+0.40}$ & $1.00 \pm 0.29$ \\
$H \rightarrow W W^{*}$ & $1.00_{-0.29}^{+0.32}$ & $0.83 \pm 0.21$ \\
$H \rightarrow b \bar{b}$ & $0.2_{-0.6}^{+0.7}$ & $0.93 \pm 0.49$ \\
$H \rightarrow \tau^{+} \tau^{-}$ & $1.09_{-0.32}^{+0.36}$ & $0.91 \pm 0.27$ \\
\hline
\end{tabular}

Table 4. Higgs signal strength data reported at ICHEP2014.

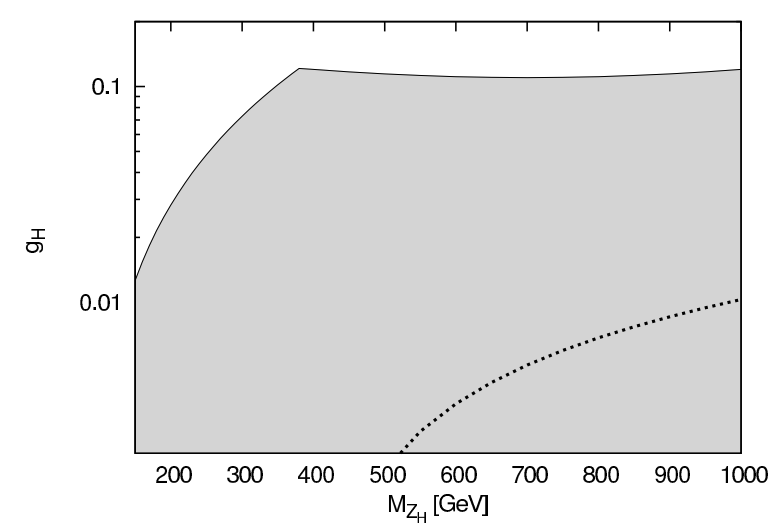

Figure 1. $M_{Z_{H}}$ and $g_{H}$ in the type-II $2 \mathrm{HDM}_{\mathrm{U}(1)}$. The dot line is the upper bound on the $\mathrm{U}(1)_{\psi}$ gauge boson, and the gray region is allowed for the $\mathrm{U}(1)_{H}\left(\equiv \mathrm{U}(1)_{b}\right)$ gauge boson.

The lower bounds on the masses of extra quarks and charged leptons are set to be $1 \mathrm{TeV}$ and $800 \mathrm{GeV}$, respectively, as discussed in the previous section. Finally, there is no bound on the mass of extra neutral leptons, $N_{i}$, where the lightest one is a candidate for CDM, $X$.

If $m_{X}$ is less than $m_{h} / 2$, the observed Higgs boson $h$ can decay to $2 X$, which contributes to the invisible decay of $h$. The bound on the invisible decay of the SM-like Higgs has been discussed in refs. [63-67]. Explicitly we assume $\mathrm{BR}(h \rightarrow$ invisibles $) \leq 0.58$. We take the mass of the extra scalars and gauge boson to be over the mass of the SM-like Higgs boson. Thus, they do not contribute to the invisible decay of $h$. Furthermore, if $m_{X}$ is lighter than the half of the $Z$-boson mass, $Z$ can also decay to $2 X$. This constraint may easily be avoided in the range $m_{X} \geq M_{Z} / 2$.

\subsection{4 $\mathrm{U}(1)_{H}$ gauge boson $Z_{H}$}

On the other hand, the $Z_{H}$ interaction is constrained by searches for a $Z^{\prime}$ boson at collider experiments. From now on we define the $\mathrm{U}(1)_{H}$ charge assignments as the leptophobic case, i.e. we consider the case $\mathrm{U}(1)_{H} \equiv \mathrm{U}(1)_{b}$. Then the $\mathrm{U}(1)_{b}$ gauge interactions of the SM particles are given by

$$
\begin{aligned}
\hat{\mathcal{L}}_{g}= & g_{H} \hat{Z}_{H}^{\mu}\left(\frac{2}{3} \overline{U_{R}^{i}} \gamma_{\mu} U_{R}^{i}-\frac{1}{3} \overline{D_{R}^{i}} \gamma_{\mu} D_{R}^{i}-\frac{1}{3} \overline{Q^{i}} \gamma_{\mu} Q^{i}+\overline{N_{R}^{i}} \gamma_{\mu} N_{R}^{i}\right)+g_{Z} \hat{Z}^{\mu} J_{\mu}^{S M} \\
& +\frac{1}{2} M_{Z_{H}}^{2} \hat{Z}_{H}^{\mu} \hat{Z}_{H \mu}+\frac{1}{2} M_{Z}^{2} \hat{Z}^{\mu} \hat{Z}_{\mu}+\Delta M^{2} \hat{Z}_{H}^{\mu} \hat{Z}_{\mu}
\end{aligned}
$$


where $M_{Z}^{2}, g_{Z}$ and $J_{\mu}^{S M}$ are the mass, the gauge coupling and the current of the $Z$ boson in the SM. The nonzero VEV of $H_{2}$ gives the mass mixing $\Delta M^{2}$ between $\hat{Z}_{H \mu}$ and $\hat{Z}_{\mu}$, so that $\hat{Z}_{H \mu}$ and $\hat{Z}_{\mu}$ are not in their mass basis. However, the mixing is strongly constrained by the EWPOs, as discussed in ref. [14], so that $\hat{Z}_{H \mu}$ and $\hat{Z}_{\mu}$ could be approximately interpreted as the gauge bosons $\left(Z_{H \mu}\right.$ and $\left.Z_{\mu}\right)$ in their mass basis. Then we can ignore the $Z_{H}$ boson couplings to the SM leptons, thereby $Z_{H}$ becoming leptophobic. The strong bounds from the Drell-Yan processes and the LEP experiment can be evaded if the mass mixing of $Z$ and $Z_{H}$ is small enough. The resonance searches for a $Z^{\prime}$ boson in the dijet and $t \bar{t}$ production also provide relevant constraints on the $Z_{H}$ boson. They give the upper bound of $g_{H}$ in the $O(100) \mathrm{GeV}$ mass region [68-71]. In figure 1, we depict the allowed region for $g_{H}$ and $M_{Z_{H}}$ in the type-II $2 \mathrm{HDM}_{\mathrm{U}(1)}$ with leptophobic $\mathrm{U}(1)_{H}\left(\equiv \mathrm{U}(1)_{b}\right)$ symmetry, which is represented by gray color. For comparison, we also show the upper bound for the $\mathrm{U}(1)_{\psi}$ gauge boson, which is represented by the dot line. The bound for the $\mathrm{U}(1)_{\psi}$ gauge boson is much stronger than that for the $\mathrm{U}(1)_{H}$ gauge boson due to the interaction with SM leptons. For the $\mathrm{U}(1)_{H}$ gauge boson, it is found that the low mass region is strictly constrained by the EWPOs, i.e. the $Z$ decay width and $\rho$ parameter. While the bound in high mass region comes mainly from the resonance searches in the dijet and $t \bar{t}$ production at hadron colliders. The allowed value for $g_{H}$ is $O(0.01)$ in the low $M_{Z_{H}}$ region and $O(0.1)$ in the high $M_{Z_{H}}$ region, respectively. We note that these upper bounds are a bit stronger than in the Type-I $2 \mathrm{HDM}_{\mathrm{U}(1)_{H}}$ because the $Z_{H}$ boson is fermiophobic in the Type-I case [14].

\section{Higgs signals at the LHC}

The SM-like Higgs boson with mass $\sim 125 \mathrm{GeV}$ was discovered at the LHC $[72,73]$. At the first stage of the measurements, the signal strengths in the Higgs decaying into two photons or $Z Z^{*}$ were slightly larger than the SM predictions. As more data were accumulated at the $8 \mathrm{TeV}$ center-of-momentum (CM) energy, however, the signal strengths became consistent with the SM predictions in each decay mode as shown in table 4. Although this consistency may imply that the discovered boson is really the SM Higgs, we still cannot rule out a possibility it could be a SM-like Higgs boson in the model with an extended Higgs sector like the 2HDM, with a small mixture from the extended Higgs sector.

In the usual Type-II 2HDM, there are two CP-even scalar bosons, while there is one more CP-even scalar boson in the $2 \mathrm{HDM}_{\mathrm{U}(1)_{H}}$. The lightest one is assumed to be the SMlike Higgs boson in this work. In both models, there is a CP-odd scalar $A$. The Yukawa couplings of the SM-like Higgs boson and extra scalar boson with the SM fermions depend on the vacuum alignment of VEVs of two Higgs doublets. In the $2 \mathrm{HDM}_{\mathrm{U}(1)_{H}}$, the $\mathrm{U}(1)_{H}$ gauge boson $Z_{H}$ and extra chiral fermions also take part in interactions. Therefore all the extra particles in $2 \mathrm{HDM}_{\mathrm{U}(1)_{H}}$ can change Higgs physics at the LHC. The charged Higgs boson can contribute to $h \rightarrow \gamma \gamma$ and $h \rightarrow Z \gamma$ at the loop level [14]. Extra charged and/or colored particles contribute to $h \rightarrow \gamma \gamma, Z \gamma$ and the $h \rightarrow g g$ at one loop level. Furthermore, the SM-like Higgs boson may decay to the extra particles, if the sum of masses of final particles are less than $m_{h}$. 

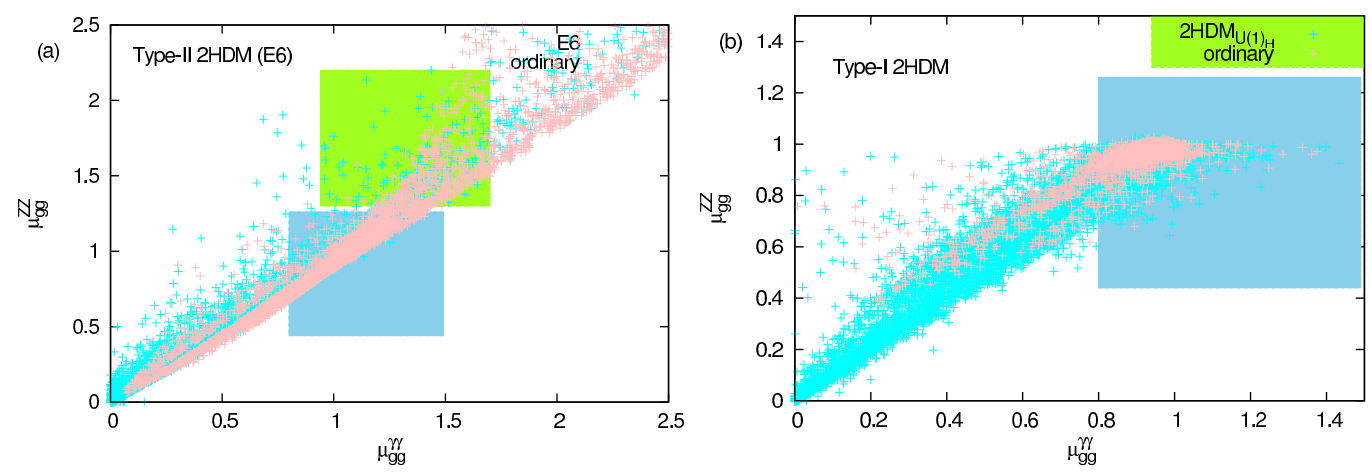

Figure 2. Signal strengths $\mu_{\gamma \gamma}^{g g}$ and $\mu_{Z Z}^{g g}$ (a) in the Type-II 2HDMs and (b) in the Type-I 2HDMs.

In figure 2, we depict the signal strengths $\mu_{\gamma \gamma}^{g g}$ and $\mu_{Z Z}^{g g}$ in the Type-II 2HDMs, which are calculated by using HDECAY [74]. We modified the original HDECAY code by modifying Higgs couplings to the SM fermions and weak gauge bosons and by including the contribution of the charged Higgs boson and extra charged fermions to the $h g g, h \gamma \gamma$, and $h Z \gamma$ vertices. We also draw the same figure in the Type-I $2 \mathrm{HDM}$ for comparison, which is based on ref. [14]. The pink points are allowed in the Type-II (-I) $2 \mathrm{HDM}_{Z_{2}}$, while the cyan points are allowed in the Type-II (-I) $2 \mathrm{HDM}_{\mathrm{U}(1)_{H}}$, respectively. The blue and green boxes are CMS and ATLAS data at $\sqrt{s}=7,8 \mathrm{TeV}$ in the $1 \sigma$ level, respectively. Explicitly, we use $\mu_{g g H}^{\gamma \gamma}=1.12_{-0.32}^{+0.37}$ and $\mu_{g g H, t \bar{t} H}^{Z Z}=0.80_{-0.36}^{+0.46}$ for the CMS data [75, 76] while $\mu_{g g F}^{\gamma \gamma}=1.32 \pm 0.38$ and $\mu_{g g F+b \bar{b} h+t \bar{t} h}^{Z Z}=1.7_{-0.4}^{+0.5}$ for the ATLAS data [77, 78].

The SM prediction for the Higgs signal strength is $\mu=1$ by definition, which is consistent with the CMS and ATLAS data within $1 \sigma$ and $2 \sigma$, respectively. This implies that new physics should not affect the Higgs signal strengths too much. In this respect, the decoupling scenario, where all the scalar bosons except the SM-like Higgs boson are heavy enough to decouple from EW physics, or the alignment scenario, where the heavy Higgs boson coupling to gauge boson is suppressed, are preferred in the 2HDMs [79, 80]. A similar situation would be true in the $2 \mathrm{HDM}_{\mathrm{U}(1)_{H}}$.

In figure 3 , we depict the allowed regions in the $((\beta-\alpha) / \pi, \tan \beta)$ planes for (a) the Type-II 2HDMs and (b) the Type-I 2HDMs, where all points are consistent with the theoretical and experimental bounds discussed in the previous sections. We note that the Higgs signal strengths $\mu_{g g}^{\gamma \gamma}$ and $\mu_{g g}^{Z Z}$ of all the points in figure 3 are consistent with the CMS data $\left(\mu_{g g}^{\gamma \gamma}\right.$ and $\left.\mu_{g g}^{Z Z}\right)$ in the $1 \sigma$ level, as shown in figure 2. If the ATLAS data or combined data of ATLAS and CMS are used, we would get similar plots.

As shown in figure 2(b), the Higgs signal strengths can reach in the following ranges: $\mu_{g g}^{\gamma \gamma} \lesssim 1.4$ and $0.4 \lesssim \mu_{g g}^{Z Z} \lesssim 1.1$ in the Type-I $2 \mathrm{HDM}_{Z_{2}}$, but $\mu_{g g}^{\gamma \gamma} \lesssim 1.4$ and $0 \lesssim \mu_{g g}^{Z Z} \lesssim 1.1$ is allowed in the Type-I $2 \mathrm{HDM}_{\mathrm{U}(1)_{H}}$. The region where $\mu_{g g}^{Z Z} \sim 0$ is allowed in the Type-I $2 \mathrm{HDM}_{\mathrm{U}(1)_{H}}$, but it is disallowed in the Type-I $2 \mathrm{HDM}_{Z_{2}}$. This is because both couplings of the SM-like Higgs boson to fermions and gauge bosons have an additional suppression factor $\cos \alpha_{1}$. That is, the rescaling factors of the SM-like Higgs boson couplings are $g_{h f f}=\cos \alpha_{1} \cos \alpha / \sin \beta$ and $g_{h V V}=\cos \alpha_{1} \sin (\beta-\alpha)$ in the Type-I $2 \mathrm{HDM}_{\mathrm{U}(1)_{H}}$. We note that the rescaling factors in the Type-I $2 \mathrm{HDM}_{Z_{2}}$ can be obtained if we set $\alpha_{1}=0$. 

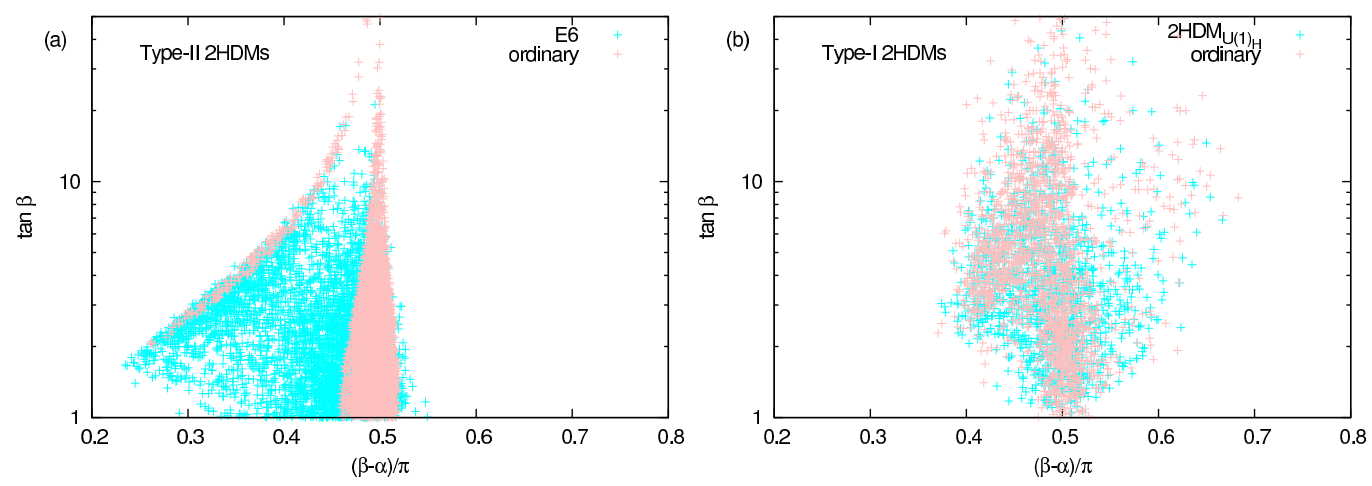

Figure 3. $(\beta-\alpha) / \pi$ vs. $\tan \beta$ (a) in the Type-II 2 HDMs and (b) in the Type-I 2 HDMs. All the points satisfy the CMS data $\left(\mu_{g g}^{\gamma \gamma}\right.$ and $\left.\mu_{g g}^{Z Z}\right)$ within $1 \sigma$ level.

On the other hand, in the Type-II case, both signal strengths $\mu_{g g}^{\gamma \gamma}$ and $\mu_{g g}^{Z Z}$ can take their values from 0 to $\sim 3$. The SM-like Higgs coupling to the SM gauge bosons are the same as in the Type-I case, but the Yukawa couplings are different. Note that the rescaling factor of the Yukawa coupling to the up-type fermions is $g_{\text {huu }}=\cos \alpha \cos \alpha_{1} / \sin \beta$, while that to the down-type fermions is $g_{h d d}=-\sin \alpha \cos \alpha_{1} / \cos \beta$.

In the Type-I case, the allowed parameter spaces in ordinary $2 \mathrm{HDM}_{Z_{2}}$ and $2 \mathrm{HDM}_{\mathrm{U}(1)_{H}}$ are rather similar. As discussed in ref. [14], $|\sin \alpha| \gtrsim 0.8$ is not allowed because the coupling $g_{h f f} \sim \cos \alpha / \sin \beta$ is small for $\tan \beta>1$. In this region $|\cos (\beta-\alpha)| \lesssim 0.4$ and the Yukawa couplings have similar values as the SM Yukawa couplings.

In the Type-II $2 \mathrm{HDM}_{Z_{2}}$, two parameter regions are allowed. One of them is $(\beta-\alpha) \sim$ $\pi / 2$ corresponding to the SM limit line, $\sin (\beta-\alpha) \sim 0$. The other branch corresponds to the line $\sin (\beta+\alpha) \sim 0$. In this branch, the Yukawa couplings of the up-type fermions are very close to the SM Yukawa couplings, while those of the down-type fermions have the opposite sign relative to the SM Yukawa couplings [81]. In the Type-II $2 \mathrm{HDM}_{\mathrm{U}(1)_{H}}$, the intermediate region between two pink branches is also allowed. This intermediate region contains the parameter space with $\sin \alpha \sim 0$. The rescaling factor of the Yukawa couplings of the up-type fermions is $\left|g_{h u \bar{u}}\right| \sim 1$, where the opposite sign is also allowed. This is because all the rescaling factors include an overall factor $\cos \alpha_{1}$. For negative $\cos \alpha_{1}$, the negative Yukawa coupling can be achieved. The rescaling factor of the down-type fermions is allowed in $\left|g_{h d \bar{d}}\right| \lesssim 1$. In particular, $\left|g_{h d \bar{d}}\right|$ may have a very small value in some points. In the analysis, we do not constrain the Yukawa couplings of the down-type fermions directly. If the Yukawa couplings of the down-type fermions are well measured in the near future, the allowed parameter spaces for $g_{h d \bar{d}}$ would strongly be constrained.

In the allowed region, both $\tan \beta$ and $\mathrm{U}(1)_{H}$ coupling are rather small: $\tan \beta \lesssim 15$ and $g_{H} \lesssim 0.13$. There are no strict bounds on the extra scalars, i.e. $m_{H, a, \tilde{h}} \geq m_{h}$ and $m_{H^{+}} \geq 360 \mathrm{GeV}$. The mass of the $\mathrm{U}(1)_{H}$ gauge boson is in the range of $m_{Z_{H}} \geq m_{h}$ and the VEV of $\Phi$ is $v_{\phi} \gtrsim 2.5 \mathrm{TeV}$. Because of the small $\mathrm{U}(1)_{H}$ gauge coupling $g_{H}$, the $Z_{H}$ boson with $100 \mathrm{GeV} \sim 1 \mathrm{TeV}$ mass can avoid the strong constraints from experiments. The mass of the dark matter candidate is in the range of $0<m_{X} \lesssim 1.2 \mathrm{TeV}$. 
In principle, our models could be distinguished from the ordinary $2 \mathrm{HDMs}$ because there exist additional particles: an additional neutral Higgs boson, a new gauge boson $Z_{H}$, and extra chiral fermions, that could be produced directly at colliders or can appear in the loop. However, note that the qualitative features in Higgs physics, in particular, the Higgs signal strengths (figure 2) are not so different between two models in the ATLAS/CMS data regions. Therefore it is not that easy to distinguish these two models only by the Higgs signal strength measured at the LHC, since the LHC data are in good agreement with the SM (see figure 2). Large deviations of the Higgs signal strengths from the SM predictions or discovery of new particles would be necessary to tell our 2HDM with local $\mathrm{U}(1)_{H}$ gauge symmetry from the usual $2 \mathrm{HDM}$ with $Z_{2}$ symmetry.

Still the detail of the model parameter space are different as shown in figure 3 . In the ordinary type-II $2 \mathrm{HDM}$, the allowed region for $\tan \beta$ and $(\beta-\alpha)$ is restricted in the two branches, while in our model, the allowed region is much broader. This is mainly due to the additional neutral Higgs boson with new mixing angles that appear in the Yukawa couplings and the Higgs couplings to the weak gauge bosons. Also the extra colored and/or charged particles cancelling the gauge anomalies generate the difference in the Higgs signal strengths through their contribution to the $h g g$ and $h \gamma \gamma$ couplings.

As we have mentioned, our models have new particles that are not present in the ordinary 2HDM: three neutral scalar bosons, a new gauge boson $Z_{H}$ and extra chiral fermions. Discovery of some of these new particles would be distinctive signatures of our model. For example, observation of extra fermions in the production/decay channels discussed in section 3 would be clear signatures of the Type-II $2 \mathrm{HDM}$ with local $\mathrm{U}(1)_{H}$ gauge symmetry.

Note that we have not imposed constraints on the dark matter candidate yet in the analysis of this section. The constraints on dark matter from the thermal relic density, direct and indirect detection of dark matter will also strongly constrain the parameter space. Still figures 2 and 3 are meaningful if we consider the model where the dark matter candidate can decay by introducing an additional scalar that couples with the dark matter candidate. Then there is no dark matter in the model so that we do not need to take into account the constraints from dark matter detection.

\section{Dark matter physics}

As we discussed in section 4 , the lightest neutral particle $X$ is a Majorana fermion and could be stable due to the remnant $Z_{2}^{\text {ex }}$ symmetry. In the mass matrix for $\left(\widetilde{\nu}_{L}, \widetilde{\nu}_{R}^{c}, n_{L}\right)$, we assume that $m_{\widetilde{e}} \gg m_{D}, m_{M}$ in order to evade the stringent constraint from the extra lepton search. In that limit, $m_{X}$ can be approximately evaluated as

$$
m_{X} \approx \frac{2 m_{D} m_{M}}{m_{\widetilde{e}}}=\frac{y^{n} y^{\prime n} v^{2}}{m_{\widetilde{e}}} \cos \beta \sin \beta,
$$

where $X$ is mostly $n_{L}$-like:

$$
X_{L} \approx n_{L}-\frac{m_{D}}{m_{\widetilde{e}}} \widetilde{\nu}_{L}-\frac{m_{M}}{m_{\widetilde{e}}} \widetilde{\nu}_{R}^{c}
$$

In order to make $m_{X}$ heavy enough, we require large Yukawa couplings, $y^{n}$ and $y^{\prime n}$ of $\sim O(1)$. 


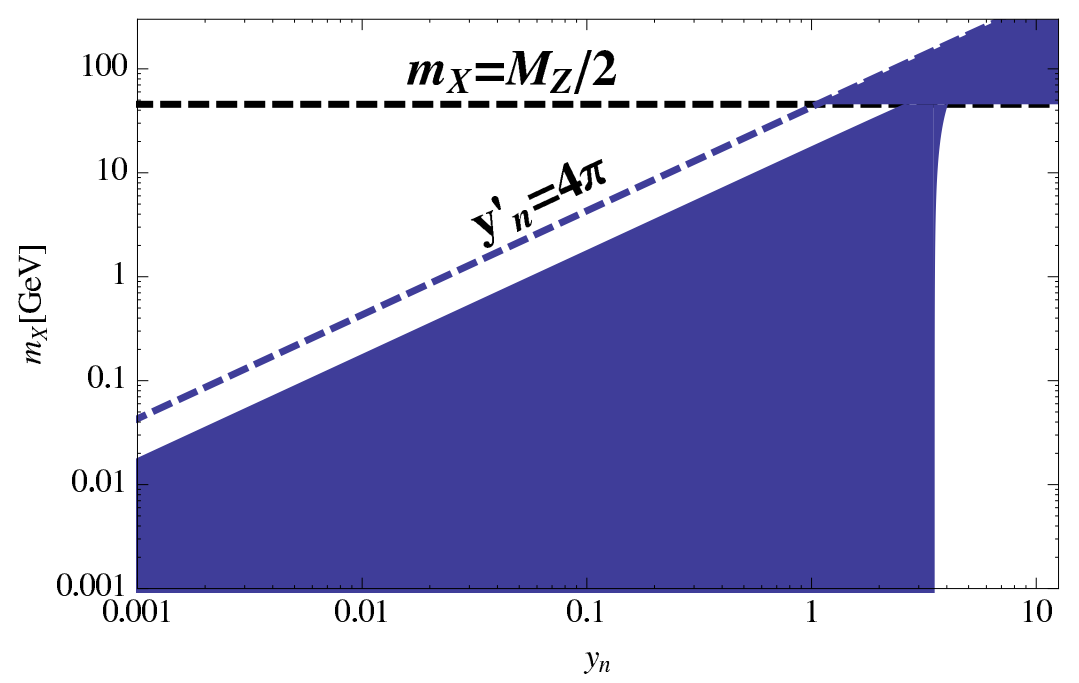

Figure 4. $y^{n}$ vs. $m_{X}$ with $\tan \beta=3$ and $m_{\widetilde{e}}=1 \mathrm{TeV}$. The blue region satisfies all the bounds on the extra particles in the text, as well as the invisible $Z$ decay $\left(y^{n} \lesssim 3\right.$ and $\left.y^{\prime n} \lesssim 1\right)$.

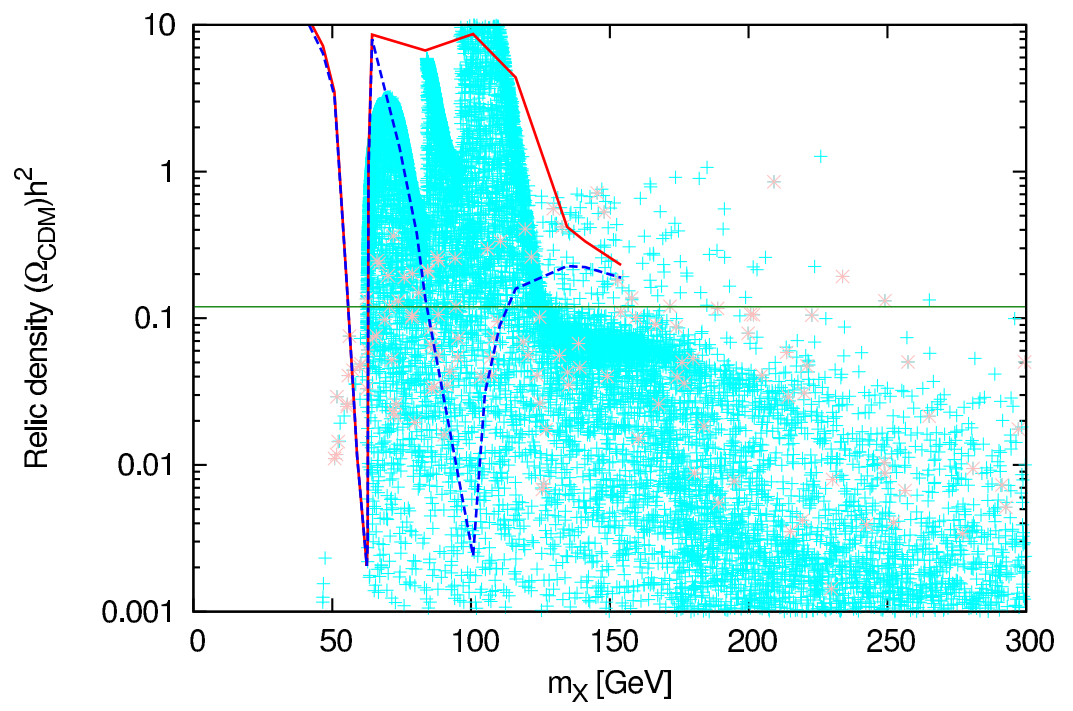

Figure 5. $m_{X}$ vs. $\Omega h^{2}$ in the decoupling limit, $\sin (\beta-\alpha)=0, \alpha_{1}=\alpha_{2}=0$. The cyan points satisfy the experimental constraints at colliders while the pink points satisfy the bound from direct detection of DM in the LUX experiment too. The red line is for $500 \mathrm{GeV} \leq m_{H, A, H^{+}} \leq 1 \mathrm{TeV}$ and $y^{\prime n}=1$, while the blue line is for $m_{A}=m_{H}=200 \mathrm{GeV}, m_{H^{+}}=360 \mathrm{GeV}$ and $y^{\prime n}=1$.

In figure 4 , the region for $m_{X}$ and $y^{n}$ is described, setting $\tan \beta=3$ and $m_{\widetilde{e}}=1 \mathrm{TeV}$. The blue region is the one allowed for the bound from the invisible decay of $Z$ boson $\left(y^{n} \lesssim 3\right.$ and $y^{\prime n} \lesssim 1$ ), which is derived from the $1 \sigma$ error of the invisible decay width of $Z$ boson [82].

$X$ could be thermally produced through the following annihilation processes: $X X \rightarrow$ $f \bar{f}, W^{+} W^{-}$, and $Z Z$. The extra fermions masses are generated by $\langle\Phi\rangle$, and could be much heavier than $X$ because of the experimental constraints, so that they have already decoupled at the freeze-out temperature of $X$. The $\mathrm{U}(1)_{H}$ gauge interaction through $Z_{H}$ emission 
may be also effective as we see in figure 1 . And $h, A, H$ exchanging in the $s$-channel are efficient in the annihilation and scattering with nuclei, because of large Yukawa coupling. However, all the cross sections are strongly suppressed by the mixing elements $U_{a b}$ 's defined in eq. (3.5) and appendix $\mathrm{A}$, so that $X$ tends to be over-produced in our universe.

In figure 5, we show the thermal relic density of $X$ in the decoupling limit where the mixing among CP-even scalars are fixed at $\sin (\beta-\alpha)=1$ and $\alpha_{1}=\alpha_{2}=0$. The cyan points satisfy the experimental constraints at colliders which have been discussed in previous sections. The pink points satisfy the LUX bound for direct detection of DM in addition to the experimental constraints at colliders.

For more concrete discussion, let us fix other parameters too. In the red line, the masses of $A, H$ and $H_{ \pm}$are within $500 \mathrm{GeV} \leq m_{H, A, H^{+}} \leq 1 \mathrm{TeV}$ and $y^{\prime n}=1$. Then the heavy scalar exchange processes are inefficient to reduce the relic density for $m_{X} \lesssim 200 \mathrm{GeV}$. The blue line corresponds to the case with $m_{A}=m_{H}=200 \mathrm{GeV}, m_{H \pm}=360 \mathrm{GeV}$, and $y^{\prime n}=1$. The green band is the observed relic density in the PLANCK experiment [83]. In both cases, only the regions around the resonances, $m_{X} \approx m_{h} / 2$ and $m_{X} \approx m_{H} / 2$, can result in the correct relic density of DM (we have calculated thermal relic density using the micrOMEGAs [84]). The spin-independent and spin-dependent direct detection cross sections of DM X on proton are estimated as $\sigma_{\mathrm{SI}}=6.54 \times 10^{-10}\left(1.98 \times 10^{-10}\right) \mathrm{pb}$ and $\sigma_{\mathrm{SD}}=2.41 \times 10^{-8}\left(1.91 \times 10^{-5}\right)$ pb at $m_{X}=55.3(83.5) \mathrm{GeV}$, where the DM density is $\Omega h^{2}=0.166(0.137)$. They are far below the current experimental bounds from the direct detection [85]. If $m_{X}$ is less than half of $m_{h}$, the SM-like Higgs can decay into a pair of DMs, and the branching ratio of the invisible decay is 0.1 at $m_{X}=55.3 \mathrm{GeV}$, which is still acceptable [65-67].

In figure 5 , there is a sharp peak around $m_{X}=50 \mathrm{GeV}$, where the relic density is highly suppressed due to the processes, $X X \rightarrow Z \rightarrow f \bar{f}(f=\mathrm{SM}$ fermions except $t)$, (the SM $Z$ boson resonance). The DM coupling with the SM $Z$ boson is generated by the $Z-Z_{H}$ mixing. At $m_{X} \approx 60 \mathrm{GeV}$, the relic density can be smaller than the current observation due to the resonance effect of the SM-like Higgs boson mediation. In the region $m_{X}>60 \mathrm{GeV}$, new resonance processes, for example, the heavy scalar $(H)$ exchange process, could contribute to decreasing the relic density. At $m_{X} \simeq 80 \mathrm{GeV}, X X \rightarrow W^{+} W^{-}$ channel is open so that the relic density could be below the observation. Surely, it strongly depends on the DM- $Z$ coupling. For a small DM- $Z$ coupling, the $X X \rightarrow W^{+} W^{-}$process is not sufficient to reduce the relic density. In that case, the relic density is higher than the current observation as shown in figure 5 .

In figure 6, we depict the DM mass vs. the velocity-averaged annihilation cross section, $\langle\sigma v\rangle$, at the halo. The calculation was carried out by using micrOMEGAs [84]. All points satisfy the collider constraints discussed in previous sections as well as the LUX bound for direct detection of DM. We impose that the thermal relic densities are below the PLANCK observation $\Omega_{\mathrm{CDM}} h^{2}=0.1199 \pm 0.0027$ with $3 \sigma$ uncertainty [83]. The horizontal line, whose value is $\langle\sigma v\rangle \simeq 3 \times 10^{-26} \mathrm{~cm}^{3} / \mathrm{s}$, corresponds to the bound from the relic density for the $s$-wave annihilation dominant case. The solid and dotted curves are the Fermi-LAT bound for the DM annihilation into $b \bar{b}$ and $W^{+} W^{-}$, respectively. 


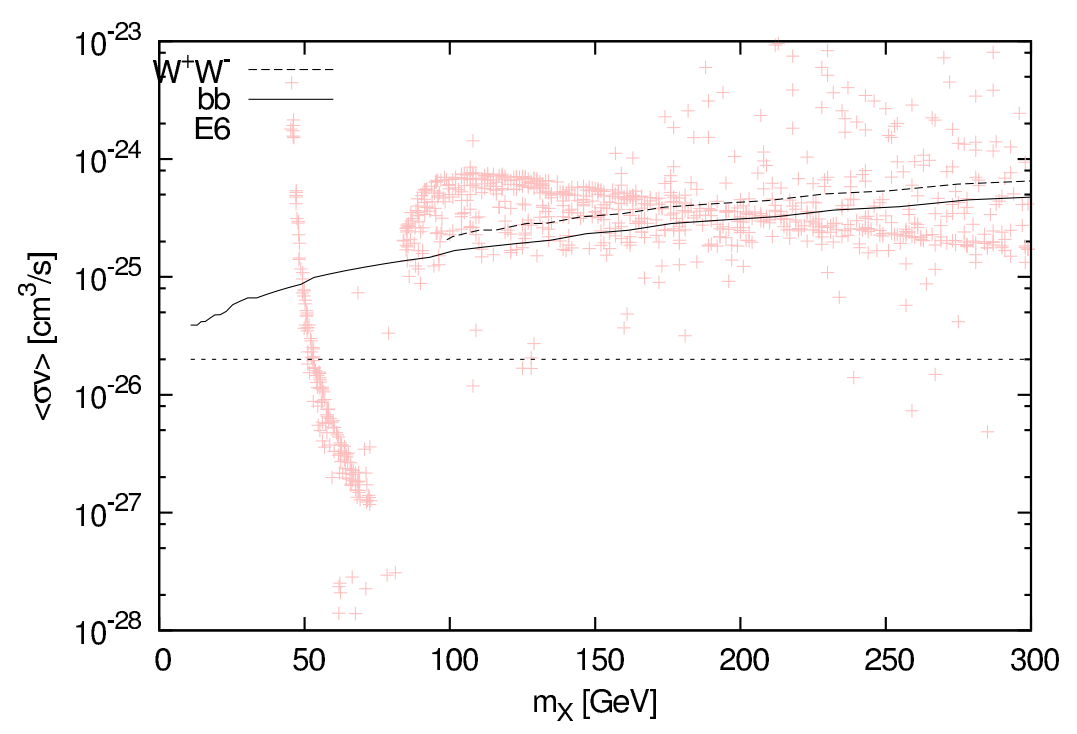

Figure 6. $m_{X}$ vs. $\langle\sigma v\rangle$ in units of $\mathrm{GeV}$ and $\mathrm{cm}^{3} / \mathrm{s}$, respectively. The pink points satisfy collider constraints and direct detection bound in the LUX experiments. The relic density is below the current observation by the PLANCK Collaboration [83].

At the $Z$-resonance region $\left(m_{X} \sim 50 \mathrm{GeV}\right)$, the indirect detection bound might severely constrain our model. The bound is assumed that $X X \rightarrow b \bar{b}$ is dominant, but in our model its contribution is about $17 \%$. The rest contribution comes from the DM annihilation into SM fermions except $b$ and $t$ pairs. This would slightly relieve the strong bound from the indirect detection of DM. As shown in figure 6, the Higgs-resonance region is less constrained because of the resonance effects coming mainly from difference between the DM velocity at the freeze-out and at the current halo. At the region $m_{X} \gtrsim m_{W}$, our model is strongly constrained by the indirect detection of DM again, but there are still some allowed regions as shown in figure 6.

\section{Summary}

In this paper, we have studied the type-II $2 \mathrm{HDM}_{\mathrm{U}(1)}$ inspired by $E_{6}$, which was proposed by the present authors a few years ago [15]. Both of the two Higgs doublets $\left(H_{1}\right.$ and $\left.H_{2}\right)$ and the SM chiral fermions are charged under $\mathrm{U}(1)_{H}$ and the theory becomes anomalous. Therefore, for the purpose of anomaly cancellation, we have introduced extra quarks and leptons, which could be derived from 27 representation together with the SM fermions, from the point of view of bottom-up approach. Unlike the fermion sector, the Higgs sector of our model has not been extended to $\mathbf{2 7}$ representation of $E_{6}$. This is the main difference between our model and the usual $E_{6}$ GUT. Still we could expect that our $2 \mathrm{HDM}_{\mathrm{U}(1)}$ is effectively realized by supersymmetric $E_{6}$ GUT. But we have simply discussed the phenomenology involving the extra Higgs doublet and fermions, assuming only one gauged $\mathrm{U}(1)_{H}$ and $Z_{2}^{\text {ex }}$ discrete symmetry which may be predicted by $E_{6}$ gauge symmetry survive at low energy. Especially, we considered the leptophobic $\mathrm{U}(1)_{H}$ charge assignments to avoid the stringent constraint from DY processes, and study the $Z_{H}$ effect on the Higgs 
and DM physics. In fact, $Z_{H}$ could be as light as $\sim O(100) \mathrm{GeV}$, but very light $Z_{H}$ is strictly constrained by the EWPOs, as we see in figure 1 . It may be difficult to draw explicit bounds on $2 \mathrm{HDMs}$ from the Higgs signal strengths at the LHC alone. But we found that the Type-II 2HDM can easily enhance or reduce the signal strength of $h \rightarrow Z Z$ and $\gamma \gamma$, because of the sensitivity to $h \rightarrow b \bar{b}$, compared with Type-I 2HDMs, so that we can expect that our Type-II $2 \mathrm{HDM}_{\mathrm{U}(1)}$ will be strictly constrained by the LHC Run-II.

Search for the extra quarks and leptons is especially important to our model. The current lower bound from the exotic fermion searches is around $800 \mathrm{GeV}$, and it will become more stringent at the LHC Run-II. We may find Majorana fermion dark matter candidate among the extra neutral particles, where the DM stability is guaranteed by $Z_{2}^{\text {ex }}$. It may be difficult to shift the mass of the DM, because of the stringent constraints on the extra charged particles and $Z_{2}^{\text {ex }} ; O(10) \mathrm{GeV}$ DM mass corresponds to $O(1)$ Yukawa couplings, $y^{n}$ and $y^{\prime n}$. If we accept such large Yukawa couplings, we can explain the correct thermal relic DM density, and escape from the strong bounds from the DM direct detections. The DM scenario predicts the invisible decay of the $125 \mathrm{GeV}$ Higgs and the branching ratio is $\sim O(0.1)$, which may be reached at the LHC. $Z_{2}^{\text {ex }}$ plays two important roles: it does not only guarantee the DM stability, but also forbid the unwanted FCNCs involving the extra fermions. In other words, we have to consider the effect of the mixing terms between the SM particles and the extra fermions as in eq. (2.8), if we cannot realize $Z_{2}^{\text {ex }}$ from $E_{6}$ gauge symmetry. It will cause problems in flavor physics, but may give some rich phenomenology in neutrino and dark matter physics. It was discussed in ref. [86] and the detail of the work is in progress.

\section{Acknowledgments}

We thank Korea Institute for Advanced Study for providing computing resources (KIAS Center for Advanced Computation Abacus System) for this work. This work was supported in part by Basic Science Research Program through the National Research Foundation of Korea (NRF) funded by the Ministry of Education Science and Technology 2011-0022996 (CY), by NRF Research Grant 2012R1A2A1A01006053 (PK and CY), and by SRC program of NRF funded by MEST (20120001176) through Korea Neutrino Research Center at Seoul National University (PK). The work of YO is supported by Grant-in-Aid for Scientific research from the Ministry of Education, Science, Sports, and Culture (MEXT), Japan, No. 23104011.

\section{A Interactions of exotic fermions}

The gauge interactions of the extra fermions are given by the following terms,

$$
\begin{aligned}
\mathcal{L}_{g}= & g_{s} G^{a \mu} \bar{q} t^{a} \gamma_{\mu} q-\frac{g^{\prime}}{3} B^{\mu} \bar{q} \gamma_{\mu} q+g_{H} \hat{Z}_{H}^{\mu}\left(Q_{q_{L}} \overline{q_{L}} \gamma_{\mu} q_{L}+Q_{q_{R}} \overline{q_{R}} \gamma_{\mu} q_{R}\right) \\
& +\frac{e}{4 c_{W} s_{W}} \hat{Z}^{\mu}\left(\left|U_{\nu_{R} a}\right|^{2}-\left|U_{\nu_{L} a}\right|^{2}\right)\left(\bar{N}_{a} \gamma_{\mu} \gamma_{5} N_{a}\right) \\
& +\sum_{(a, b)=(a, a+1)} \frac{e}{2 c_{W} s_{W}} \hat{Z}^{\mu}\left\{\operatorname{Re}\left(U_{\nu_{R} a}^{*} U_{\nu_{R} b}\right)-\operatorname{Re}\left(U_{\nu_{L} a}^{*} U_{\nu_{L} b}\right)\right\}\left(\bar{N}_{a} \gamma_{\mu} \gamma_{5} N_{b}\right)
\end{aligned}
$$




$$
\begin{aligned}
& +\sum_{(a, b)=(a, a+1)} \frac{i e}{2 c_{W} s_{W}} \hat{Z}^{\mu}\left\{\operatorname{Im}\left(U_{\nu_{L} a}^{*} U_{\nu_{L} b}\right)-\operatorname{Im}\left(U_{\nu_{R} a}^{*} U_{\nu_{R} b}\right)\right\}\left(\bar{N}_{a} \gamma_{\mu} N_{b}\right) \\
& -\frac{e\left(c_{W}^{2}-s_{W}^{2}\right)}{2 c_{W} s_{W}} \hat{Z}^{\mu} \overline{\tilde{e}} \gamma_{\mu} \widetilde{e}-e A^{\mu} \overline{\widetilde{e}} \gamma_{\mu} \widetilde{e} \\
& +\frac{e}{s_{W} 2 \sqrt{2}} W^{\mu \dagger}\left\{\left(U_{\nu_{L} a}+U_{\nu_{R} a}^{*}\right) \overline{\widetilde{e}} \gamma_{\mu} N_{a}-\left(U_{\nu_{L} a}-U_{\nu_{R} a}^{*}\right) \overline{\widetilde{e}} \gamma_{\mu} \gamma_{5} N_{a}\right\} \\
& +\frac{e}{s_{W} 2 \sqrt{2}} W^{\mu}\left\{\left(U_{\nu_{L} a}^{*}+U_{\nu_{R} a}\right) \overline{N_{a}} \gamma_{\mu} \widetilde{e}-\left(U_{\nu_{L} a}^{*}-U_{\nu_{R} a} a\right) \bar{N}_{a} \gamma_{\mu} \gamma_{5} \widetilde{e}\right\} \\
& +\frac{g_{H}}{2} \hat{Z}_{H}^{\mu}\left(Q_{\widetilde{e}_{R}}\left|U_{\nu_{R} a}\right|^{2}-Q_{\widetilde{e}_{L}}\left|U_{\nu_{L} a}\right|^{2}-Q_{n_{L}}\left|U_{n_{L} a}\right|^{2}\right)\left(\bar{N}_{a} \gamma_{\mu} \gamma_{5} N_{a}\right) \\
& +g_{H} \hat{Z}_{H}^{\mu} \sum_{(a, b)=(a, a+1)}\left\{Q_{\widetilde{e}_{R}} \operatorname{Re}\left(U_{\nu_{R} a}^{*} U_{\nu_{R} b}\right)-Q_{\widetilde{e}_{L}} \operatorname{Re}\left(U_{\nu_{L} a}^{*} U_{\nu_{L} b}\right)-Q_{n_{L}} \operatorname{Re}\left(U_{n_{L} a}^{*} U_{n_{L} b}\right)\right\}\left(\bar{N}_{a} \gamma_{\mu} \gamma_{5} N_{b}\right) \\
& -i g_{H} \hat{Z}_{H}^{\mu} \sum_{(a, b)=(a, a+1)}\left\{Q_{\widetilde{e}_{R}} \operatorname{Im}\left(U_{\nu_{R} a}^{*} U_{\nu_{R} b}\right)-Q_{\widetilde{e}_{L}} \operatorname{Im}\left(U_{\nu_{L} a}^{*} U_{\nu_{L} b}\right)-Q_{n_{L}} \operatorname{Im}\left(U_{n_{L} a}^{*} U_{n_{L} b}\right)\right\}\left(\bar{N}_{a} \gamma_{\mu} N_{b}\right),
\end{aligned}
$$

where $\{(a, b)\}=\{(1,2),(2,3),(3,1)\}$ and $(3,4)=(3,1)$ are defined. The flavor index is omitted assuming the masses are degenerate and the mixing, $U_{a b}$, is defined in eq. (3.5).

The Yukawa couplings involving neutral fermions may be relevant to dark matter physics:

$$
\begin{aligned}
\mathcal{L}_{Y}= & -\frac{m_{\widetilde{e}}}{v_{\Phi}}\left(U_{\nu_{R} a} U_{\nu_{L} b}\right) \Phi \overline{N^{a}} P_{L} N^{b}-\frac{m_{D}}{v \cos \beta}\left(U_{\nu_{R} a} U_{n_{L} b}\right) H_{1}^{0} \overline{N^{a}} P_{L} N^{b}-\frac{m_{M}}{v \sin \beta}\left(U_{\nu_{L} a} U_{n_{L} b}\right) H_{2}^{0} \overline{N^{a}} P_{L} N^{b} \\
& -\frac{m_{D} \sqrt{2}}{v \cos \beta} U_{n_{L} b} H_{1}^{-} \overline{\widetilde{e}} P_{L} N^{b}-\frac{m_{M} \sqrt{2}}{v \sin \beta} U_{n_{L} b} H_{2}^{+} \overline{\widetilde{e}^{c}} P_{L} N^{b}+h . c . .
\end{aligned}
$$

$H_{1,2}^{0}$ and $H_{1,2}^{ \pm}$are the neutral and charged components of the two Higgs doublets and they generally mix with each other as discussed in ref. [14].

\section{B Contribution of the extra lepton to the vacuum polarization}

Simply assuming that the masses are degenerate among the generations, the corrections of the vacuum polarizations are given by

$$
\begin{aligned}
\Delta \Pi_{W W}\left(q^{2}\right)= & \frac{e^{2} N_{F}}{8 s_{W}^{2}} \sum_{a}\left\{\left|U_{\nu_{L} a}+U_{\nu_{R} a}^{*}\right|^{2} \Pi_{V}\left(q^{2}, m_{\widetilde{e}}, m_{a}\right)+\left|U_{\nu_{L} a}-U_{\nu_{R} a}^{*}\right|^{2} \Pi_{A}\left(q^{2}, m_{\widetilde{e}}, m_{a}\right)\right\}, \\
\Delta \Pi_{Z Z}\left(q^{2}\right)= & \frac{N_{F} e^{2}\left(c_{W}^{2}-s_{W}^{2}\right)^{2}}{4 c_{W}^{2} s_{W}^{2}} \Pi_{V}\left(q^{2}, m_{\widetilde{e}}, m_{\tilde{e}}\right) \\
& +\sum_{a} \frac{N_{F} e^{2}}{8 c_{W}^{2} s_{W}^{2}}\left(\left|U_{\nu_{R} a}\right|^{2}-\left|U_{\nu_{L} a}\right|^{2}\right)^{2} \Pi_{A}\left(q^{2}, m_{a}, m_{a}\right) \\
& +\sum_{(a, b)=(a, a+1)} \frac{N_{F} e^{2}}{4 c_{W}^{2} s_{W}^{2}}\left\{R e\left(U_{\nu_{R} a}^{*} U_{\nu_{R} b}\right)-\operatorname{Re}\left(U_{\nu_{L} a}^{*} U_{\nu_{L} b}\right)\right\}^{2} \Pi_{A}\left(q^{2}, m_{a}, m_{b}\right) \\
& +\sum_{(a, b)=(a, a+1)} \frac{N_{F} e^{2}}{4 c_{W}^{2} s_{W}^{2}}\left\{\operatorname{Im}\left(U_{\nu_{R} a}^{*} U_{\nu_{R} b}\right)-\operatorname{Im}\left(U_{\nu_{L} a}^{*} U_{\nu_{L} b} b\right\}^{2} \Pi_{V}\left(q^{2}, m_{a}, m_{b}\right)\right. \\
\Delta \Pi_{Z \gamma}\left(q^{2}\right)= & N_{F} \frac{e^{2}\left(c_{W}^{2}-s_{W}^{2}\right)}{2 c_{W} s_{W}} \Pi_{V}\left(q^{2}, m_{\widetilde{e}}, m_{\widetilde{e}}\right) \\
\Delta \Pi_{\gamma \gamma}\left(q^{2}\right)= & N_{F} e^{2} \Pi_{V}\left(q^{2}, m_{\widetilde{e}}, m_{\widetilde{e}}\right)
\end{aligned}
$$


where $\Pi^{V}\left(\Pi^{A}\right)$ is the vacuum polarization of vector currents (axial currents) and defined as

$$
\begin{aligned}
& \Pi^{V}\left(q^{2}, m_{1}, m_{2}\right)=\frac{-1}{8 \pi^{2}}\left\{\left(q^{2}-\left(m_{1}-m_{2}\right)^{2}\right) B_{0}\left(q^{2}, m_{1}^{2}, m_{2}^{2}\right)+4 B_{22}\left(q^{2}, m_{1}^{2}, m_{2}^{2}\right)-A\left(m_{1}^{2}\right)-A\left(m_{2}^{2}\right)\right\}, \\
& \Pi^{A}\left(q^{2}, m_{1}, m_{2}\right)=\frac{-1}{8 \pi^{2}}\left\{\left(q^{2}-\left(m_{1}+m_{2}\right)^{2}\right) B_{0}\left(q^{2}, m_{1}^{2}, m_{2}^{2}\right)+4 B_{22}\left(q^{2}, m_{1}^{2}, m_{2}^{2}\right)-A\left(m_{1}^{2}\right)-A\left(m_{2}^{2}\right)\right\} .
\end{aligned}
$$

The explicit expressions of the functions $B_{0}, B_{22}$ and $A$ and the contribution of the Majorana particles can be found in ref. [46, 87].

Open Access. This article is distributed under the terms of the Creative Commons Attribution License (CC-BY 4.0), which permits any use, distribution and reproduction in any medium, provided the original author(s) and source are credited.

\section{References}

[1] G.C. Branco et al., Theory and phenomenology of two-Higgs-doublet models, Phys. Rept. 516 (2012) 1 [arXiv:1106.0034] [INSPIRE].

[2] G. Bélanger, B. Dumont, U. Ellwanger, J.F. Gunion and S. Kraml, Global fit to Higgs signal strengths and couplings and implications for extended Higgs sectors, Phys. Rev. D 88 (2013) 075008 [arXiv: 1306.2941] [INSPIRE].

[3] C.-W. Chiang and K. Yagyu, Implications of Higgs boson search data on the two-Higgs doublet models with a softly broken $Z_{2}$ symmetry, JHEP 07 (2013) 160 [arXiv: 1303.0168] [INSPIRE].

[4] B. Grinstein and P. Uttayarat, Carving out parameter space in type-II two Higgs doublets model, JHEP 06 (2013) 094 [Erratum ibid. 09 (2013) 110] [arXiv: 1304.0028] [INSPIRE].

[5] A. Celis, V. Ilisie and A. Pich, LHC constraints on two-Higgs doublet models, JHEP 07 (2013) 053 [arXiv: 1302.4022] [INSPIRE].

[6] H.S. Cheon and S.K. Kang, Constraining parameter space in type-II two-Higgs doublet model in light of a $126 \mathrm{GeV}$ Higgs boson, JHEP 09 (2013) 085 [arXiv: 1207.1083] [INSPIRE].

[7] S. Chang et al., Comprehensive study of two Higgs doublet model in light of the new boson with mass around $125 \mathrm{GeV}$, JHEP 05 (2013) 075 [arXiv: 1210.3439] [INSPIRE].

[8] S. Chang et al., Two Higgs doublet models for the LHC Higgs boson data at $\sqrt{s}=7$ and $8 \mathrm{TeV}$, JHEP 09 (2014) 101 [arXiv: 1310.3374] [INSPIRE].

[9] A. Celis, V. Ilisie and A. Pich, Towards a general analysis of LHC data within two-Higgs-doublet models, JHEP 12 (2013) 095 [arXiv: 1310.7941] [INSPIRE].

[10] N. Craig, J. Galloway and S. Thomas, Searching for signs of the second Higgs doublet, arXiv: 1305.2424 [INSPIRE].

[11] P.M. Ferreira, R. Guedes, M.O.P. Sampaio and R. Santos, Wrong sign and symmetric limits and non-decoupling in 2HDMs, JHEP 12 (2014) 067 [arXiv: 1409.6723] [INSPIRE].

[12] J. Song and Y.W. Yoon, Gigantic diphoton rate of heavy Higgs bosons in the aligned two Higgs doublet models with small $\tan \beta$, arXiv:1412.5610 [INSPIRE].

[13] S.L. Glashow and S. Weinberg, Natural conservation laws for neutral currents, Phys. Rev. D 15 (1977) 1958 [INSPIRE].

[14] P. Ko, Y. Omura and C. Yu, Higgs phenomenology in type-I 2 HDM with $\mathrm{U}(1)_{H}$ Higgs gauge symmetry, JHEP 01 (2014) 016 [arXiv:1309.7156] [INSPIRE]. 
[15] P. Ko, Y. Omura and C. Yu, A resolution of the flavor problem of two Higgs doublet models with an extra $\mathrm{U}(1)_{H}$ symmetry for Higgs flavor, Phys. Lett. B 717 (2012) 202 [arXiv: 1204.4588] [INSPIRE].

[16] P. Ko, Y. Omura and C. Yu, Top forward-backward asymmetry and the CDF Wjj excess in leptophobic U(1)' flavor models, Phys. Rev. D 85 (2012) 115010 [arXiv:1108.0350] [INSPIRE].

[17] P. Ko, Y. Omura and C. Yu, Chiral U(1) flavor models and flavored Higgs doublets: the top FB asymmetry and the $W j j$, JHEP 01 (2012) 147 [arXiv:1108.4005] [INSPIRE].

[18] P. Ko, Y. Omura and C. Yu, Dark matter and dark force in the type-I inert 2 HDM with local $\mathrm{U}(1)_{H}$ gauge symmetry, JHEP 11 (2014) 054 [arXiv: 1405.2138] [INSPIRE].

[19] S. Baek, P. Ko and W.-I. Park, Singlet portal extensions of the standard seesaw models to a dark sector with local dark symmetry, JHEP 07 (2013) 013 [arXiv:1303.4280] [INSPIRE].

[20] S. Baek, P. Ko and W.-I. Park, Hidden sector monopole, vector dark matter and dark radiation with Higgs portal, JCAP 10 (2014) 067 [arXiv:1311.1035] [INSPIRE].

[21] P. Ko and Y. Tang, Self-interacting scalar dark matter with local $Z_{3}$ symmetry, JCAP 05 (2014) 047 [arXiv: 1402.6449] [INSPIRE].

[22] P. Ko and Y. Tang, $\nu \Lambda M D M$ : a model for sterile neutrino and dark matter reconciles cosmological and neutrino oscillation data after BICEP2, Phys. Lett. B 739 (2014) 62 [arXiv: 1404.0236] [INSPIRE].

[23] S. Baek, P. Ko and W.-I. Park, Local $Z_{2}$ scalar dark matter model confronting galactic GeV-scale $\gamma$-ray and muon $(g-2)$, arXiv: 1407.6588 [INSPIRE].

[24] P. Ko and Y. Tang, AMS02 positron excess from decaying fermion DM with local dark gauge symmetry, Phys. Lett. B 741 (2015) 284 [arXiv:1410.7657] [INSPIRE].

[25] S.M. Barr, Effects of extra light $Z$ bosons in unified and superstring models, Phys. Rev. Lett. 55 (1985) 2778 [INSPIRE].

[26] D. London and J.L. Rosner, Extra gauge bosons in E $E_{6}$, Phys. Rev. D 34 (1986) 1530 [INSPIRE].

[27] J.L. Hewett and T.G. Rizzo, Low-energy phenomenology of superstring inspired $E_{6}$ models, Phys. Rept. 183 (1989) 193 [inSPIRE].

[28] S.F. King, S. Moretti and R. Nevzorov, Theory and phenomenology of an exceptional supersymmetric standard model, Phys. Rev. D 73 (2006) 035009 [hep-ph/0510419] [INSPIRE].

[29] K.S. Babu, C.F. Kolda and J. March-Russell, Leptophobic U(1)s and the $R_{b}-R_{c}$ crisis, Phys. Rev. D 54 (1996) 4635 [hep-ph/9603212] [INSPIRE].

[30] T.G. Rizzo, Gauge kinetic mixing in the $E_{6}$ SSM, Phys. Rev. D 85 (2012) 055010 [arXiv:1201.2898] [INSPIRE].

[31] CMS collaboration, Search for resonances in the dilepton mass distribution in pp collisions at $\sqrt{s}=8 \mathrm{TeV}$, CMS-PAS-EXO-12-061, CERN, Geneva Switzerland (2012).

[32] J.L. Rosner, Prominent decay modes of a leptophobic Z', Phys. Lett. B 387 (1996) 113 [hep-ph/9607207] [INSPIRE].

[33] K. Leroux and D. London, Flavor changing neutral currents and leptophobic Z' gauge bosons, Phys. Lett. B 526 (2002) 97 [hep-ph/0111246] [INSPIRE]. 
[34] M.R. Buckley, D. Hooper and J.L. Rosner, A leptophobic $Z^{\prime}$ and dark matter from grand unification, Phys. Lett. B 703 (2011) 343 [arXiv:1106.3583] [INSPIRE].

[35] C.-W. Chiang, T. Nomura and K. Yagyu, Phenomenology of $E_{6}$-inspired leptophobic $Z^{\prime}$ boson at the LHC, JHEP 05 (2014) 106 [arXiv: 1402.5579] [INSPIRE].

[36] CMS collaboration, Search for electroweak production of charginos, neutralinos and sleptons using leptonic final states in pp collisions at $8 \mathrm{TeV}$, CMS-PAS-SUS-13-006, CERN, Geneva Switzerland (2013).

[37] ATLAS collaboration, Search for direct-slepton and direct-chargino production in final states with two opposite-sign leptons, missing transverse momentum and no jets in $20 \mathrm{fb}^{-1}$ of pp collisions at $\sqrt{s}=8 \mathrm{TeV}$ with the ATLAS detector, ATLAS-CONF-2013-049, CERN, Geneva Switzerland (2013).

[38] ATLAS collaboration, Search for strongly produced supersymmetric particles in decays with two leptons at $\sqrt{s}=8 \mathrm{TeV}$, in First Large Hadron Collider physics conference, Barcelona Spain May 13-18 2013 [ATLAS-CONF-2013-089].

[39] S. Kanemura, T. Kasai and Y. Okada, Mass bounds of the lightest CP even Higgs boson in the two Higgs doublet model, Phys. Lett. B 471 (1999) 182 [hep-ph/9903289] [INSPIRE].

[40] A.G. Akeroyd, A. Arhrib and E.-M. Naimi, Note on tree level unitarity in the general two Higgs doublet model, Phys. Lett. B 490 (2000) 119 [hep-ph/0006035] [INSPIRE].

[41] I.F. Ginzburg and I.P. Ivanov, Tree-level unitarity constraints in the most general 2HDM, Phys. Rev. D 72 (2005) 115010 [hep-ph/0508020] [InSPIRE].

[42] M.E. Peskin and T. Takeuchi, Estimation of oblique electroweak corrections, Phys. Rev. D 46 (1992) 381 [INSPIRE].

[43] Particle Data Group collaboration, J. Beringer et al., Review of particle physics (RPP), Phys. Rev. D 86 (2012) 010001 [inSPIRE].

[44] M. Baak et al., The electroweak fit of the standard model after the discovery of a new boson at the LHC, Eur. Phys. J. C 72 (2012) 2205 [arXiv:1209.2716] [INSPIRE].

[45] M. Baak and R. Kogler, The global electroweak standard model fit after the Higgs discovery, arXiv:1306.0571 [INSPIRE].

[46] H.-J. He, N. Polonsky and S.-F. Su, Extra families, Higgs spectrum and oblique corrections, Phys. Rev. D 64 (2001) 053004 [hep-ph/0102144] [INSPIRE].

[47] S. Kanemura, Y. Okada, H. Taniguchi and K. Tsumura, Indirect bounds on heavy scalar masses of the two-Higgs-doublet model in light of recent Higgs boson searches, Phys. Lett. B 704 (2011) 303 [arXiv:1108.3297] [INSPIRE].

[48] W. Grimus, L. Lavoura, O.M. Ogreid and P. Osland, A precision constraint on multi-Higgs-doublet models, J. Phys. G 35 (2008) 075001 [arXiv:0711.4022] [InSPIRE].

[49] W. Grimus, L. Lavoura, O.M. Ogreid and P. Osland, The oblique parameters in multi-Higgs-doublet models, Nucl. Phys. B 801 (2008) 81 [arXiv:0802.4353] [INSPIRE].

[50] ALEPH, DELPHI, L3, OPAL and LEP collaborations, G. Abbiendi et al., Search for charged Higgs bosons: combined results using LEP data, Eur. Phys. J. C 73 (2013) 2463 [arXiv: 1301.6065] [INSPIRE]. 
[51] ATLAS collaboration, Search for charged Higgs bosons decaying via $H^{ \pm} \rightarrow \tau^{ \pm} \nu$ in fully hadronic final states using pp collision data at $\sqrt{s}=8 \mathrm{TeV}$ with the ATLAS detector, JHEP 03 (2015) 088 [arXiv: 1412.6663] [INSPIRE].

[52] CMS collaboration, Search for charged Higgs bosons with the $H^{+} \rightarrow \tau \nu$ decay channel in the fully hadronic final state at $\sqrt{s}=8 \mathrm{TeV}$, CMS-PAS-HIG-14-020, CERN, Geneva Switzerland (2014).

[53] T. Hermann, M. Misiak and M. Steinhauser, $\bar{B} \rightarrow X_{s} \gamma$ in the two Higgs doublet model up to next-to-next-to-leading order in QCD, JHEP 11 (2012) 036 [arXiv:1208.2788] [INSPIRE].

[54] Belle collaboration, I. Adachi et al., Evidence for $B^{-} \rightarrow \tau^{-} \bar{\nu}_{\tau}$ with a hadronic tagging method using the full data sample of Belle, Phys. Rev. Lett. 110 (2013) 131801 [arXiv: 1208.4678] [INSPIRE].

[55] BABAr collaboration, B. Aubert et al., A search for $B^{+} \rightarrow \ell^{+} \nu_{\ell}$ recoiling against $B^{-} \rightarrow D^{0} \ell^{-} \bar{\nu} X$, Phys. Rev. D 81 (2010) 051101 [arXiv:0912.2453] [INSPIRE].

[56] Belle collaboration, K. Hara et al., Evidence for $B^{-} \rightarrow \tau^{-} \bar{\nu}$ with a semileptonic tagging method, Phys. Rev. D 82 (2010) 071101 [arXiv: 1006.4201] [InSPIRE].

[57] A. Barroso, P.M. Ferreira, R. Santos, M. Sher and J.P. Silva, $2 H D M$ at the LHC - the story so far, arXiv: 1304.5225 [INSPIRE].

[58] ATLAS collaboration, Search for the neutral Higgs bosons of the minimal supersymmetric standard model in pp collisions at $\sqrt{s}=7 \mathrm{TeV}$ with the ATLAS detector, JHEP 02 (2013) 095 [arXiv: 1211.6956] [INSPIRE].

[59] CMS collaboration, Search for MSSM neutral Higgs bosons decaying to tau pairs in pp collisions, CMS-PAS-HIG-13-021, CERN, Geneva Switzerland (2013).

[60] BABAR collaboration, J.P. Lees et al., Evidence for an excess of $\bar{B} \rightarrow D^{(*)} \tau^{-} \bar{\nu}_{\tau}$ decays, Phys. Rev. Lett. 109 (2012) 101802 [arXiv:1205.5442] [INSPIRE].

[61] P. Ko, Y. Omura and C. Yu, $B \rightarrow D^{(*)} \tau \nu$ and $B \rightarrow \tau \nu$ in chiral $\mathrm{U}(1)^{\prime}$ models with flavored multi Higgs doublets, JHEP 03 (2013) 151 [arXiv:1212.4607] [INSPIRE].

[62] ATLAS collaboration, Measurements of the properties of the Higgs-like boson in the four lepton decay channel with the ATLAS detector using $25 \mathrm{fb}^{-1}$ of proton-proton collision data, ATLAS-CONF-2013-013, CERN, Geneva Switzerland (2013).

[63] CMS collaboration, Search for invisible decays of Higgs bosons in the vector boson fusion and associated ZH production modes, Eur. Phys. J. C 74 (2014) 2980 [arXiv:1404.1344] [INSPIRE].

[64] ATLAS collaboration, Search for invisible decays of a Higgs boson produced in association with a Z boson in ATLAS, Phys. Rev. Lett. 112 (2014) 201802 [arXiv:1402.3244] [INSPIRE].

[65] J.R. Espinosa, M. Muhlleitner, C. Grojean and M. Trott, Probing for invisible Higgs decays with global fits, JHEP 09 (2012) 126 [arXiv:1205.6790] [INSPIRE].

[66] G. Bélanger, B. Dumont, U. Ellwanger, J.F. Gunion and S. Kraml, Global fit to Higgs signal strengths and couplings and implications for extended Higgs sectors, Phys. Rev. D 88 (2013) 075008 [arXiv:1306.2941] [INSPIRE]. 
[67] S. Choi, S. Jung and P. Ko, Implications of LHC data on 125 GeV Higgs-like boson for the standard model and its various extensions, JHEP 10 (2013) 225 [arXiv:1307.3948] [INSPIRE].

[68] CDF collaboration, T. Aaltonen et al., Search for new particles decaying into dijets in proton-antiproton collisions at $\sqrt{s}=1.96 \mathrm{TeV}$, Phys. Rev. D 79 (2009) 112002 [arXiv:0812.4036] [INSPIRE].

[69] CMS collaboration, Search for narrow resonances using the dijet mass spectrum in $p p$ collisions at $\sqrt{s}=8 \mathrm{TeV}$, Phys. Rev. D 87 (2013) 114015 [arXiv:1302.4794] [inSPIRE].

[70] CDF collaboration, Search for resonant $t \bar{t}$ production in the semi-leptonic decay mode using the full CDF data set, CDF/PUB/EXOTIC/CDFR/10927, Fermilab, Batavia U.S.A. (2012).

[71] ATLAS collaboration, Search for $t \bar{t}$ resonances in the lepton plus jets final state with ATLAS using $4.7 \mathrm{fb}^{-1}$ of pp collisions at $\sqrt{s}=7 \mathrm{TeV}$, Phys. Rev. D 88 (2013) 012004 [arXiv: 1305.2756] [INSPIRE].

[72] ATLAS collaboration, Observation of a new particle in the search for the standard model Higgs boson with the ATLAS detector at the LHC, Phys. Lett. B 716 (2012) 1 [arXiv: 1207.7214] [INSPIRE].

[73] CMS collaboration, Observation of a new boson at a mass of $125 \mathrm{GeV}$ with the CMS experiment at the LHC, Phys. Lett. B 716 (2012) 30 [arXiv:1207.7235] [INSPIRE].

[74] A. Djouadi, J. Kalinowski and M. Spira, HDECAY: a program for Higgs boson decays in the standard model and its supersymmetric extension, Comput. Phys. Commun. 108 (1998) 56 [hep-ph/9704448] [INSPIRE].

[75] CMS collaboration, Observation of the diphoton decay of the Higgs boson and measurement of its properties, Eur. Phys. J. C 74 (2014) 3076 [arXiv: 1407.0558] [INSPIRE].

[76] CMS collaboration, Measurement of the properties of a Higgs boson in the four-lepton final state, Phys. Rev. D 89 (2014) 092007 [arXiv: 1312.5353] [InSPIRE].

[77] ATLAS collaboration, Measurements of Higgs boson production and couplings in the four-lepton channel in pp collisions at center-of-mass energies of 7 and $8 \mathrm{TeV}$ with the ATLAS detector, Phys. Rev. D 91 (2015) 012006 [arXiv:1408.5191] [INSPIRE].

[78] ATLAS collaboration, Measurement of Higgs boson production in the diphoton decay channel in pp collisions at center-of-mass energies of 7 and $8 \mathrm{TeV}$ with the ATLAS detector, Phys. Rev. D 90 (2014) 112015 [arXiv:1408.7084] [INSPIRE].

[79] M. Carena, I. Low, N.R. Shah and C.E.M. Wagner, Impersonating the standard model Higgs boson: alignment without decoupling, JHEP 04 (2014) 015 [arXiv:1310.2248] [INSPIRE].

[80] P.S.B. Dev and A. Pilaftsis, Maximally symmetric two Higgs doublet model with natural standard model alignment, JHEP 12 (2014) 024 [arXiv:1408.3405] [INSPIRE].

[81] B. Dumont, J.F. Gunion, Y. Jiang and S. Kraml, Constraints on and future prospects for two-Higgs-doublet models in light of the LHC Higgs signal, Phys. Rev. D 90 (2014) 035021 [arXiv: 1405.3584] [INSPIRE].

[82] Particle Data Group collaboration, K.A. Olive et al., Review of particle physics, Chin. Phys. C 38 (2014) 090001 [INSPIRE].

[83] Planck collaboration, P.A.R. Ade et al., Planck 2013 results. XVI. Cosmological parameters, Astron. Astrophys. 571 (2014) A16 [arXiv:1303.5076] [INSPIRE]. 
[84] G. Bélanger, F. Boudjema, A. Pukhov and A. Semenov, MicrOMEGAs 3 : a program for calculating dark matter observables, Comput. Phys. Commun. 185 (2014) 960 [arXiv: 1305.0237] [INSPIRE].

[85] LUX collaboration, D.S. Akerib et al., First results from the LUX dark matter experiment at the Sanford Underground Research Facility, Phys. Rev. Lett. 112 (2014) 091303 [arXiv: 1310.8214] [INSPIRE].

[86] P. Ko, Y. Omura and C. Yu, Multi-Higgs doublet models with local $\mathrm{U}(1)_{H}$ gauge symmetry and neutrino physics therein, AIP Conf. Proc. 1604 (2014) 210 [INSPIRE].

[87] B.A. Kniehl and H.-G. Kohrs, Oblique radiative corrections from Majorana neutrinos, Phys. Rev. D 48 (1993) 225 [INSPIRE]. 